\title{
Multi-source Spatiotemporal Tracking using Sparse Large Aperture Arrays
}

\author{
Y. I. Kamil, Member, IEEE, and A. Manikas, Senior Member, IEEE,
}

\begin{abstract}
In this paper, a multi-source tracking technique is proposed using a sparse large aperture array of passive sensors of known geometry. Firstly a novel spherical-spatiotemporal-statespace model is introduced incorporating target ranges, directions and Doppler effects in conjunction with the array geometry. Subsequently, this array of sensors is integrated with an Extended Kalman Filter (EKF), defined as the Arrayed EKF, to track the trajectory of multiple mobile sources. In addition, a recursive lower bound on the performance of the proposed tracking method is obtained based on the Posterior Cramer-Rao Bound (Posterior CRB). Computer simulation studies show that the proposed approach can track the locations of sources, as these move in space, with a very high accuracy.
\end{abstract}

Index Terms-Array Processing, Spatiotemporal, Tracking, Extended Kalman Filter, Posterior Cramer-Rao Bound

\section{NOTATION}

\begin{tabular}{|c|c|}
\hline$A, a$ & Scalar \\
\hline$\underline{A}, \underline{a}$ & Column vector \\
\hline$\overline{\mathbb{A}}, \overline{\mathbf{A}}$ & Matrix \\
\hline$(\cdot)^{T},(\cdot)^{H}$ & Transpose, Hermitian transpose \\
\hline$\odot, \oslash$ & Hadamard product, division \\
\hline$\otimes$ & Kronecker product \\
\hline$\otimes$ & Khatri-Rao row-wise product \\
\hline $\exp (\underline{A})$ & $\begin{array}{l}\text { Element by element exponential of the } \\
\text { vector } \underline{A}\end{array}$ \\
\hline$\underline{A}^{b}$ & Element by element power of the vector $\underline{A}$ \\
\hline$|A|$ & Absolute value of the complex scalar $A$ \\
\hline$\|\underline{A}\|$ & Euclidian norm of the vector $\underline{A}$ \\
\hline $\mathcal{E}\{\cdot\}$ & Expectation operator \\
\hline $\operatorname{vec}(\mathbb{A})$ & $\begin{array}{l}\text { Vectorization of the matrix } \mathbb{A} \text { by stacking } \\
\text { its columns }\end{array}$ \\
\hline $\mathbb{I}_{N}$ & Identity matrix of dimensions $N \times N$ \\
\hline$\underline{1}_{N}$ & Column vector of $N$ ones \\
\hline $\mathcal{R}$ & set of real numbers \\
\hline $\mathcal{C}$ & set of complex numbers \\
\hline
\end{tabular}

Manuscript received ??; revised ??. This work was supported by the Data \& Information Fusion Defence Technology Center (DIF DTC) UK, under the project "Mobile Sentinel Wireless Sensor Networks".

Y I Kamil was with Imperial College London and is now with WesternGeco Ltd., Schlumberger House, Buckingham Gate, Gatwick, RH6 0NZ United Kingdom (email: YIzzeldin@slb.com). A. Manikas is with the Department of Electrical and Electronic Engineering, Imperial College London, London, SW7 2AZ, UK (email: a.manikas@imperial.ac.uk).

\section{INTRODUCTION}

$\mathbf{M}$ ULTIPLE source/target ${ }^{1}$ tracking is an important element of any surveillance and monitoring system that is required to incessantly monitor the spatial features of sources. For example, tracking the trajectories of multiple sources can assist in effectively managing their resources in space [1]. Other applications include air traffic control [2], missile defence [3], asset tracking [4], mobile communications [5] and wireless sensor networks [6][7].

Intuitively, the problem of tracking multiple sources can be solved by dividing the tracking period into sufficiently small time intervals over which sources can be assumed to be stationary. In this case, the tracking problem can be solved as a recursive localization problem. Here, location estimates made in a given time interval by employing any high resolution localization algorithm are propagated to the next interval to provide initial location information of the sources current position. However, in general, localization algorithms don't account for source mobility. As a result they will begin to exhibit poor performance in the presence of fast moving sources when it can no longer be assumed that the source is stationary over the observation interval due to the spread spatial spectrum created by the source motion [8]. An alternative approach to tracking in the literature is to introduce a state space model to represent target motion dynamics. Using the state space model, various Bayesian approaches are employed to estimate the locations of targets on a snapshot by snapshot basis [9][10]. These algorithms provide a better performance at the cost of an increased computational complexity.

Another issue in source tracking is concerned with the multi-source case in determining which location estimates correspond to which source over the different observation intervals. If there are $M$ targets and $M$ corresponding direction estimates, there will be $M$ ! possible combinations in which these can be assigned to one another. This is known as the association problem. Several approaches in the literature have been developed to tackle this issue. Standard methods involve searching over all possible combinations [11]-[14]. However, these methods are clearly prohibited by their large computational complexity. Other methods avoid the association problem, providing a natural assignment of direction estimates to sources. This is achieved for example by assuming that that distinct transmit powers can be used to identify the sources over the tracking period [15][16]. However, this assumption is

\footnotetext{
${ }^{1}$ Note that, in this paper, the terms "transmitters", "emitters", "sources" and "targets" are considered equivalent and are used interchangeably.
} 
not realistic in many practical scenarios.

Regardless of the tracking algorithm used, one would intuitively expect degradation in the tracking performance where the DOA trajectories of sources cross. This is a well-documented problem discussed in [8][9][10] and can frequently happen in practical scenarios since the DOAs of sources can be equal even though their trajectories are not actually crossing (i.e. they are at the same direction but different ranges). Moreover, most of the approaches in the literature do not consider the Doppler effects of the moving sources which can provide valuable information about their radial velocities.

This paper is concerned with the multiple source tracking problem which is solved using a large aperture array of passive sensors with known geometry connected with fiber, wire line or wireless links. Note that this is distinctly different to the active tracking ([17], [18], [19], [20]) or active localisation (e.g. [21]) approaches discussed extensively for example in the "radar" literature. For instance, in [17], a multi-target tracking approach is proposed that employs a radar network that uses a new particle-filter tracking method with global feedback. MIMO radar is also an "active" echo tracking system where a number of Tx-antennas transmit independent but known signals and the $\mathrm{Rx}$-antennas receive the various echoes . For example, in [18], Bayesian estimators (including Kalman and "particle" filters) are proposed for tracking in MIMO radar. Note that both [17] and [18] use underlying statistical models that are different from the array processing modelling proposed in this paper. Furthermore, in [22] MIMO radar that employs multiple, spatially distributed transmitters and receivers is presented. In particular, high resolution target localisation using MIMO radar with widely separated antennas is investigated - under the assumption that transmitted waveforms are orthogonal and that orthogonality is maintained even for different mutual delays. Note that [22] is concerned with target detection and localisation and the tracking problem can be solved as a recursive localization problem.

In this paper, under a large array aperture, it can be assumed that sources will lie in the near-far field of the array and hence undergo a spherical wave rather than a plane wave propagation model. In this case, the spherical wave manifold vector describes the array response and is a function of range as well as direction. The Doppler effects of moving sources are also considered by employing a Tapped Delay Line (TDL) at each array element. This allows the concept of the spatiotemporal spherical wave manifold to be developed. Based on this spatiotemporal manifold, a state space model is derived and used in conjunction with an Arrayed Extended Kalman Filter (Arrayed EKF) to track the range and direction of the targets. The advantage of this approach is threefold. Firstly, the dynamics governing the motion of sources can be incorporated in the tracking algorithm as prior knowledge of the state vector which can, therefore, improve the estimation accuracy. Secondly, since the Arrayed EKF depends on a single snapshot rather than constructing a sample covariance matrix, the required computation is relatively low and the adaptation to moving sources is fast. Therefore, the proposed algorithm can even be considered as suitable to wireless sensor networks which are characterized by their stringent requirement of computational power-efficiency. Finally, the proposed approach avoids the association problem because the recursive nature of the state space model preserves the natural ordering of the source locations.

In time invariant statistical models, a commonly used lower bound is the Cramer-Rao bound (CRB), given by the inverse of the Fisher information matrix [23]-[26]. This places a lower bound on the variance of any unbiased parameter estimator. If the state vector in the state space model is random, a lower bound that is analogous to the CRB can be used for similar time varying statistical systems [27]. This bound is usually referred to as the Posterior CRB. In this work, the Posterior $\mathrm{CRB}$ matrix is derived and some interesting properties of it are established.

The remainder of this paper is organized as follows: In Section II, a novel formulation of the multi-source tracking problem is presented using a large aperture array of passive sensors. Here, the received signal vector is modelled using the concept of the spatiotemporal manifold vector under spherical wave propagation leading to a novel state space model being derived. In Section III, the Arrayed Extended Kalman Filter (Arrayed EKF) is applied to this state space model to track multiple sources in the near-far field of the array. Following this, in Section IV, the Posterior CRB for the proposed spatiotemporal tracking approach, describing the lower bound on its performance, is formulated. In Section $\mathrm{V}$, computer simulation studies are presented showing the performance of the proposed algorithm. The paper is concluded in Section VI.

\section{Problem Formulation}

Consider a fully calibrated large aperture sparse array of $N$ passive sensors with locations in Cartesian coordinates given by the matrix $\mathbf{r} \in \mathcal{R}^{3 \times N}$ with respect to the system origin. That is

$$
\mathbf{r}=\left[\underline{r}_{1}, \underline{r}_{2}, \ldots, \underline{r}_{N}\right]=\left[\underline{r}_{x}, \underline{r}_{y}, \underline{r}_{z}\right]^{T}
$$

where $\underline{r}_{i} \in \mathcal{R}^{3 \times 1}$ for $i=1,2, \cdots, N$ denotes the location of the $i^{\text {th }}$ element in the array and $\underline{r}_{x}, \underline{r}_{y}, \underline{r}_{z} \in \mathcal{R}^{N \times 1}$ denote the vectors describing the $x, y$ and $z$ coordinates of the array elements. Without loss of generality, assume the first array sensor is located at the array reference point (i.e., $\underline{r}_{1}=[0,0,0]^{T}$ ). Consider that the array operates in the presence of $M$ narrowband sources/targets/transmitters with common carrier frequency $F_{c}$ where the $i^{t h}$ source transmits an unknown message signal $m_{i}(t)$ defined as

$$
m_{i}(t)=\sum_{k=-\infty}^{\infty} m_{i}[k] c\left(t-k T_{c s}\right)
$$

where

$$
k T_{c s} \leq t<(k+1) T_{c s}
$$

Here, $m_{i}[k] \forall k \in \mathcal{N}$ is a data stream of random channel symbols with zero mean and unity variance. The symbol duration is denoted by $T_{c s}$ and $c(t)$ denotes the pulse shaping waveform. It is assumed that different symbols in the data stream are completely uncorrelated such that

$$
\mathcal{E}\left\{m_{i}[p] m_{i}[q]\right\}=\delta_{p q}
$$




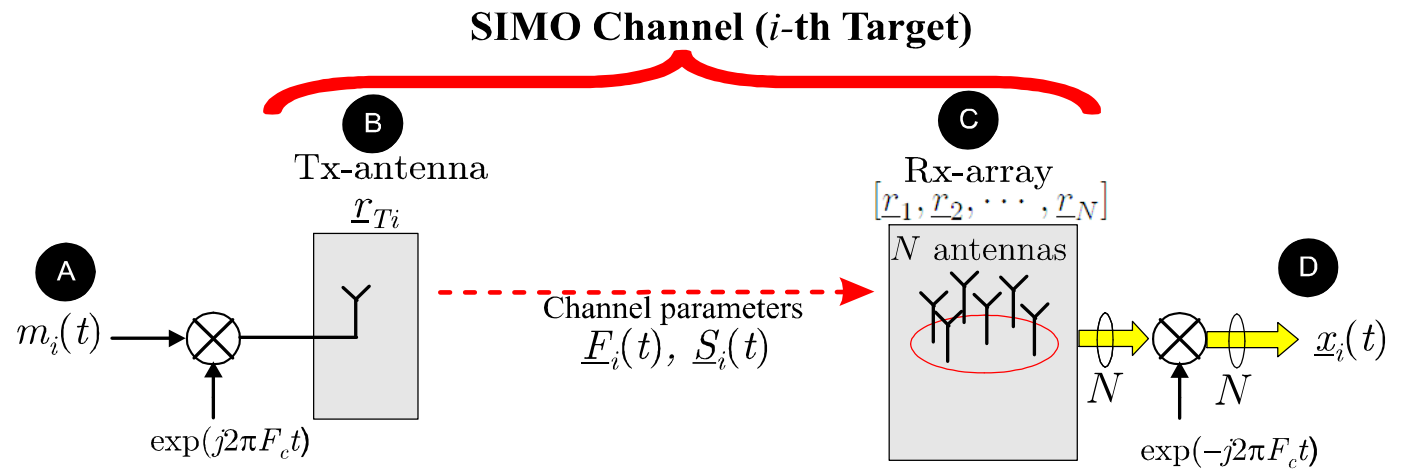

Fig. 1: SIMO Wireless System Model

The unknown message $m_{i}(t)$ of Equation 2 is shown at point $\mathrm{A}$ in Figure 1. This figure shows the SIMO (singleinput multiple-output) wireless channel associated with the $i^{t h}$ source/target where its Tx is located at $\underline{r}_{T i}(t)$ (see point B). Note that $\underline{r}_{T i}(t)$ is a time varying function with azimuth $\theta_{i}(t)$, elevation $\phi_{i}(t)$ and range $\rho_{i}(t)$ measured with respect to the array reference point. That is,

$$
\underline{r}_{T i}(t)=\rho_{i}(t) \underline{u}\left(\theta_{i}(t), \phi_{i}(t)\right) \in \mathcal{R}^{3 \times 1}
$$

Furthermore, $\theta_{i}(t)$ is measured anticlockwise with respect to the positive $\mathrm{x}$-axis. In Equation 5,

$$
\underline{u}_{i}(t) \triangleq \underline{u}\left(\theta_{i}(t), \phi_{i}(t)\right)=\left[\begin{array}{c}
\cos \theta_{i}(t) \cos \phi_{i}(t) \\
\sin \theta_{i}(t) \cos \phi_{i}(t) \\
\sin \phi_{i}(t)
\end{array}\right] \in \mathcal{R}^{3 \times 1}
$$

denotes the unity norm vector pointing towards the direction of the target $\left(\theta_{i}(t), \phi_{i}(t)\right)$. The wavenumber vector $\underline{k}_{i}(t) \in \mathcal{R}^{3 \times 1}$ which also points towards the direction $\left(\theta_{i}(t), \phi_{i}(t)\right)$ can then be defined as,

$$
\underline{k}_{i}(t)=\frac{2 \pi F_{c}}{c} \cdot \underline{u}_{i}(t)
$$

where $c$ is the propagation velocity of the transmitted signal. In this paper it is assumed with no loss of generality that the array elements and sources lie in the same $\mathcal{R}^{2}$ space (i.e. $\underline{r}_{z}=\underline{0}_{N}$ and $\left.\phi_{i}(t)=0\right)$. In addition, it is assumed that the targets move at a constant speed over a given observation interval subject to minor Brownian disturbances [9] acting on their heading directions. Finally, it is assumed that the array aperture ${ }^{2}$ is sufficiently large such that the targets lie in the near-far field of the array and thus undergo spherical wave propagation.

In this section the array spatiotemporal received vectorsignal (observation model) is first derived in Sections II.A to relate the signals received at the array to the unknown time-varying parameters (e.g. the source location) and the wide aperture array geometry. Following this, a novel spatiotemporal-state-space model is presented in Sections II.B and II-C that incorporates the observation model as well as the dynamics governing the motion of sources in the estimation process. Note that these two models (observation and state-

\footnotetext{
${ }^{2}$ Since the array of sensors has a "large aperture", which is defined as the largest distance between any two elements in the array, the proposed mode utilizes spherical wave propagation in conjuction with the spatiotemporal array response (spatiotemporal array manifold vector).
}

space models) form the basis of the novel Arrayed-EKF tracking algorithm presented in Section III.

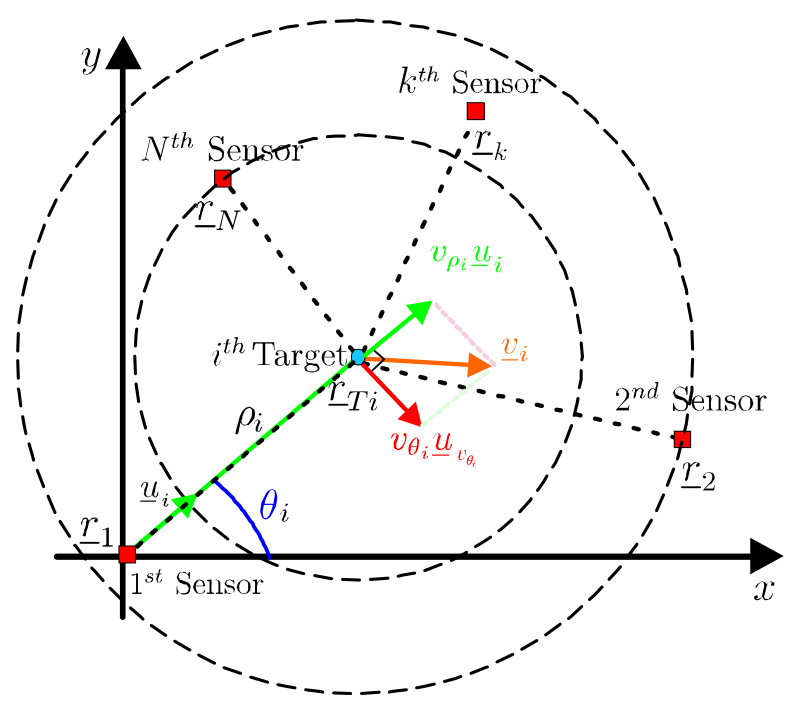

Fig. 2: A representative example in $\mathcal{R}^{2}$ space of spherical wave propagation of the $i^{t h}$ source/target (located at $\underline{r}_{T i}$ ), to the $N$ sensors in a wide aperture array. The velocity vector of the target $\underline{v}_{i}$ is split into its radial and orthoradial terms denoted by $v_{\rho_{i}} \underline{u}_{i}$ and $v_{\theta_{i}} \underline{u}_{v_{\theta_{i}}}$ respectively. The range $\rho_{i}$ and direction $\theta_{i}$ of the target are measured with respect to the array reference point.

\section{A. Spatiotemporal Array: Received Signal Model}

With reference to Figure 2, consider that the large aperture array defined in Equation 1 is tracking the $i^{\text {th }}$ target defined in Equation 5 which is transmitting the unknown message signal $m_{i}(t)$ in Equation 2. Based again on the SIMO structure shown in Figure 1, the baseband signal vector $\underline{x}_{i}(t) \in C^{N \times 1}$ at point $\mathrm{D}$ received by the passive array of sensors at point $\mathrm{C}$ can be modelled, under the wideband assumption, as

$$
\underline{x}_{i}(t)=\underline{S}_{i}(t) \odot \underline{F}_{i}(t) \odot \underline{\mathrm{m}}_{i}(t)+\underline{n}(t)
$$

where the vector $\underline{S}_{i}(t) \triangleq \underline{S}\left(\theta_{i}(t), \rho_{i}(t)\right) \in \mathcal{C}^{N \times 1}$ represents the $N$-dimensional array manifold (array response) vector, $\underline{F}_{i}(t) \in \mathcal{C}^{N \times 1}$ encompasses the effect of Doppler shift due to the velocity of the target, the vector $\underline{\mathrm{m}}_{i}(t) \in \mathcal{C}^{N \times 1}$ denotes 


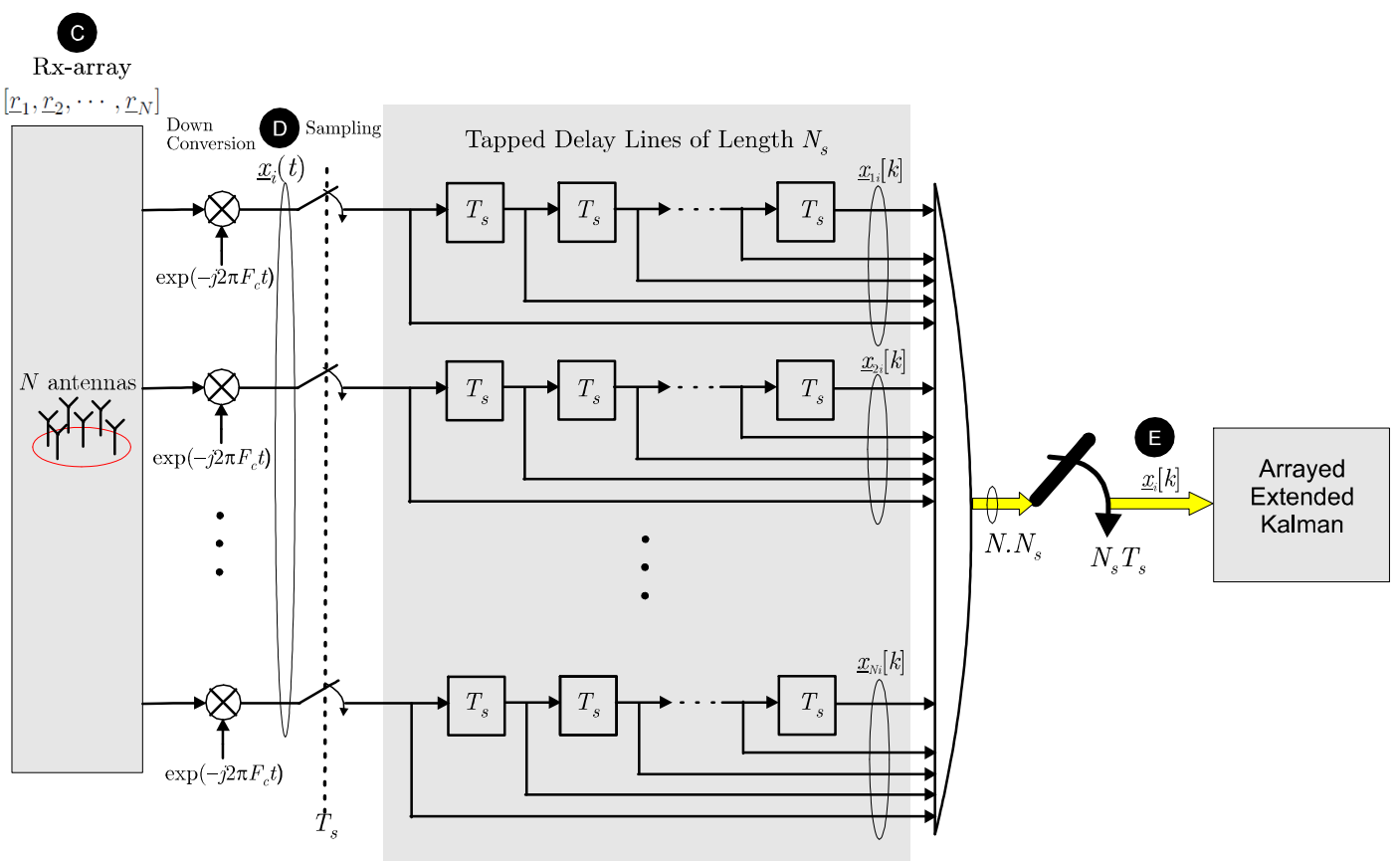

Fig. 3: Processing the received signal vector in a bank of $N$ tapped delay lines (TDLs), each of length $N_{s}$.

the delayed version of the message signals received at the array elements and $\underline{n}(t) \in \mathcal{C}^{N \times 1}$ models the noise. In this paper, $\underline{n}(t) \in \mathcal{C}^{N \times 1}$ is assumed to be zero mean additive white Gaussian noise with covariance matrix

$$
\mathbb{R}_{n n}=\sigma_{n}^{2} \mathbb{I}_{N} \in \mathcal{C}^{N \times N}
$$

where $\sigma_{n}^{2}$ denotes the noise power. However, it should be noted that the assumption of spatially white noise is not a restriction on the proposed approach providing the noise covariance can be found (up to an unknown scalar). Furthermore, the vector $\underline{\mathrm{m}}_{i}(t) \in \mathcal{C}^{N \times 1}$ is defined as

$$
\underline{\mathrm{m}}_{i}(t)=\left[m_{i}\left(t-\tau_{1 i}\right), m_{i}\left(t-\tau_{2 i}\right), \cdots, m_{N}\left(t-\tau_{N i}\right)\right]^{T}
$$

where $m_{i}\left(t-\tau_{k i}\right)$ denotes the message signal received by the $k^{t h}$ array element and is a delayed version of the message signal $m_{i}(t)$. Note that if the message signal is slowly varying as it propagates through the array (i.e. narrowband assumption) then

$$
\underline{\mathrm{m}}_{i}(t)=\underbrace{m_{i}(t-\tau)}_{\triangleq m_{i}(t)} \underline{1}_{N}
$$

The time varying array response vector $S_{i}(t)$ can be expressed using the spherical wave array manifold vector. Dropping the parameter $t$ for notational convenience and assuming omnidirectional array elements,

$$
\underline{S}_{i}(t)=\rho_{i}^{a} \cdot \underline{\rho}_{i}^{-a} \odot \exp \left(-j \frac{2 \pi F_{c}}{c}\left(\rho_{i} \underline{1}_{N}-\underline{\rho}_{i}\right)\right),
$$

where $a$ is the path loss exponent (assumed known) and $\rho_{i}$ (see Figure 2) describes the range from the $i^{t h}$ target to the array reference point, i.e. the first array element. Furthermore, $\rho_{i} \in \mathcal{R}^{N \times 1}$ is related to the unknown range $\rho_{i}$ and direction $\bar{\theta}_{i}^{i}$ of the emitting source with respect to the array reference point and the known array geometry as

$$
\underline{\rho}_{i}=\sqrt{\rho_{i}^{2} \cdot \underline{1}_{N}+\underline{r}_{x}^{2}+\underline{r}_{y}^{2}+\underline{r}_{z}^{2}-\frac{\rho_{i} c}{\pi F_{c}}\left[\underline{r}_{x}, \underline{r}_{y}, \underline{0}_{N}\right] \underline{k}\left(\theta_{i}\right)} .
$$

Note that in Equations 12 and 13, $\rho_{i}$ and $\theta_{i}$ are functions of time $t$. In this section, the parameter $t$ has been dropped from $\rho_{i}$ and $\theta_{i}$ for notational convenience.

With reference to Equation 8, the vector $\underline{F}_{i}(t)$ models the effect of Doppler shift due to the velocity vector of the $i^{t h}$ target $\underline{v}_{i} \in \mathcal{R}^{3 \times 1}$ and can be expressed as

$$
\underline{F}_{i}(t)=\exp \left(\mathrm{j} \frac{2 \pi F_{c}}{c} t\left(\rho_{i} v_{\rho_{i}} \underline{1}_{N}-\left[\underline{r}_{x}, \underline{r}_{y}, \underline{0}_{N}\right] \underline{v}_{i}\right) \odot \underline{\rho}_{i}^{-1}\right)
$$

where $v_{\rho_{i}}$ denotes the radial velocity of the target. With reference to Figure 2, the velocity vector $\underline{v}_{i}$ can be split into its radial component denoted by $v_{\rho_{i}} \underline{u}_{i} \in \mathcal{R}^{3 \times 1}$ as well as its orthoradial component denoted by $v_{\theta_{i}} \underline{u}_{v_{\theta_{i}}} \in \mathcal{R}^{3 \times 1}$ as

$$
\underline{v}_{i}=v_{\rho_{i}} \underline{u}_{\rho_{\rho_{i}}}+v_{\theta_{i}} \underline{u}_{v_{\theta_{i}}}
$$

noting that $\underline{u}_{v_{\theta_{i}}}$ is a unit norm vector. Note that the vector $\underline{F}_{i}(t)$ is a function of the range $\rho_{i}$ and direction $\theta_{i}$ of the emitting source with respect to the array reference point as well as the array geometry.

With reference to Figure 3, now consider that the $N$ dimensional received signal vector $\underline{x}_{i}(t)$ is sampled at point $\mathrm{D}$ with a period $T_{s}$ and passed through a bank of $N$ tapped delay lines (TDLs). Consider that each TDL has a length $N_{s}$ where $T_{c s}=N_{s} T_{s}$. The output of the TDLs are concatenated and sampled with a period $N_{s} T_{s}$ to create the discretized signal vector $\underline{x}_{i}[k] \in \mathcal{C}^{N N_{s} \times 1}$ at point $\mathrm{E}$ (see Figure 3 ) expressed as

$$
\underline{x}_{i}[k]=\left[\underline{x}_{1 i}^{T}[k], \underline{x}_{2 i}^{T}[k], \ldots, \underline{x}_{N i}^{T}[k]\right]^{T}
$$

where $\underline{x}_{j i}[k]=\underline{x}_{j i}\left(k T_{c s}\right) \in \mathcal{C}^{N_{s} \times 1}$ is the output from the $j^{t h}$ 
array elements TDL. The discretized signal vector $\underline{x}_{i}[k]$ can be modelled, under the "wideband" (WB-A) or "narrowband" (NB-A) assumption, as

$$
\begin{array}{ll}
\text { WB-A } & : \quad \underline{x}_{i}[k]=\underline{\mathfrak{h}}_{i}[k] \odot\left(\underline{\mathrm{m}}_{i}[k] \otimes \underline{1}_{N_{s}}\right)+\underline{n}[k] \\
\text { NB-A } & : \quad \underline{x}_{i}[k]=\underline{\mathfrak{h}}_{i}[k] m_{i}[k]+\underline{n}[k]
\end{array}
$$

where $\underline{\mathfrak{h}}_{i}[k] \triangleq \mathfrak{h}\left(\rho_{i}, v_{\rho_{i}}, \theta_{i}, v_{\theta_{i}}\right) \in \mathcal{C}^{N N_{s} \times 1}$ is defined as the Spherical SpatioTemporal ARray (Spherical STAR) manifold vector due to the $i^{t h}$ target transmission. This is a function of the array geometry (known) and the unknown time varying source parameters which are defined by the vector $\underline{z}_{i}[k] \in$ $\mathcal{R}^{4 \times 1}$ where

$$
\underline{z}_{i}[k] \triangleq\left[\rho_{i}[k], v_{\rho_{i}}[k], \theta_{i}[k], v_{\theta_{i}}[k]\right]^{T}
$$

For the remainder of this section, the time parameter $k$ will be dropped from the notation of these source parameters (given in Equation 18) for notational convenience.

Thus, in Equation 17, the vector $\underline{\mathfrak{h}}_{i}[k]$ can be modelled as

$$
\underline{\mathfrak{h}}_{i}[k]=\left(\underline{S}_{i}[k] \otimes \underline{1}_{N_{s}}\right) \odot \underline{\mathcal{F}}_{i}[k] .
$$

Here, $\underline{S}_{i}$ is the spherical wave manifold vector defined in Equation 12 which in this paper is assumed to change at a much slower rate than the vector $\underline{F}_{i}$. In addition, $\underline{\mathcal{F}}_{i}[k] \in$ $\mathcal{C}^{N N_{s} \times 1}$ encompasses the discretized effect of Doppler shift and is given by

$$
\begin{aligned}
& \underline{\mathcal{F}}_{i}[k] \triangleq \operatorname{vec}\{\underbrace{\left[\underline{F}_{i}(1), \quad \underline{F}_{i}(2), \quad \cdots, \quad \underline{F}_{i}\left(N_{s}\right)\right]^{T}}_{N \times N_{s}}\} \\
& =v e c\left\{\exp \{\mathrm{j} \frac{2 \pi F_{c}}{c} \underbrace{\left[\left(\rho_{i} v_{\rho_{i}} \underline{1}_{N}-\mathbf{r}^{T} \underline{v}_{i}\right) \odot \underline{\rho}_{i}^{-1}\right]}_{N \times 1}\right. \\
& \underbrace{\left[\begin{array}{ccc}
1, & 2, \quad \ldots, & N_{s}
\end{array}\right]}_{1 \times N_{s}} T_{s}\}\}
\end{aligned}
$$

which is a function of discretized time. Note that when the $i^{t h}$ source is in the far field of the array, the vector $\underline{\mathcal{F}}_{i}[k]$ is no longer a function of the source range, direction and the array geometry. In this case, the Spherical STAR vector is reduced to a similar structure to that of the Space-Time-Doppler (STD) manifold vector introduced ${ }^{3}$ in [28].

Some applications of this model include battlefield communications and cellular communications. In the former, sensor nodes operating as a wireless array can be attached to vehicles or human beings (e.g. soldiers) to enable parasitic mobility, controlled solely by the attached mobile hosts. In the latter it is useful for tracking mobile sources/signals. For instance, using a number of base stations as a large aperture array and transmitting messages from these mobile sources, the problem of locating and tracking these sources is inherently a near-far

\footnotetext{
${ }^{3}$ With the exception that the information signal here is not assumed to have any special temporal characteristics while in [28] the signal is assumed to be a DS-CDMA signal.
}

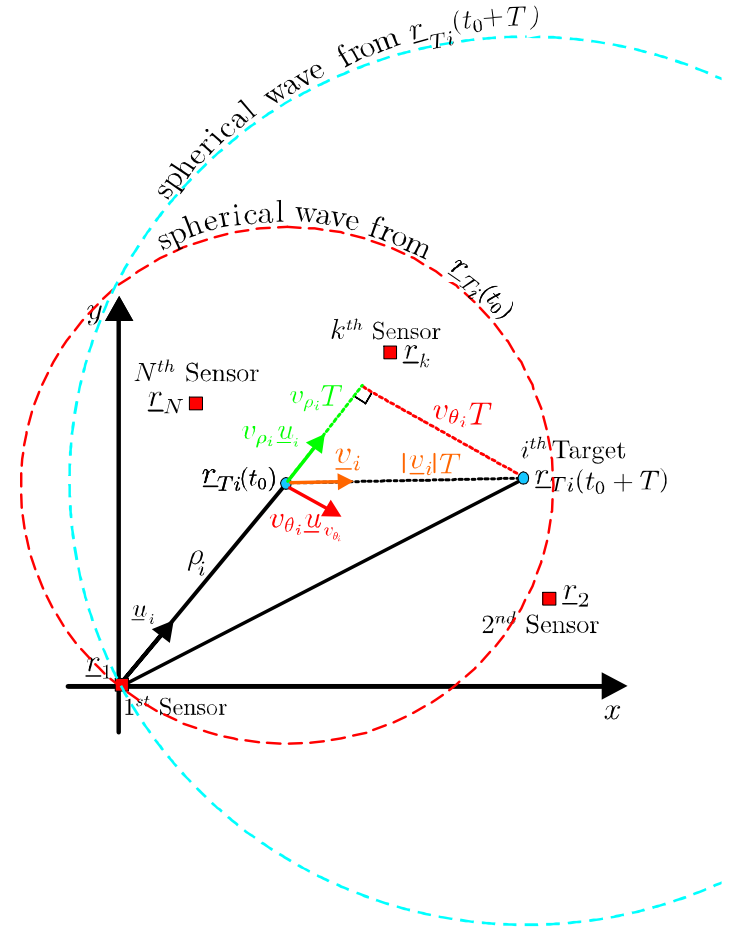

Fig. 4: A representative example in $\mathcal{R}^{2}$ space of the $i^{\text {th }}$ source/target, moving on a straight line constant velocity track relative to the array reference point over $T$ seconds. The track distance $\left|\underline{v}_{i}\right| T$ and the corresponding radial and orthoradial distances, $v_{\rho_{i}} T$ and $v_{\theta_{i}} T$, are also shown.

field problem that requires successive estimation of range and bearing angles.

\section{B. The Constant Velocity Motion State Model}

With reference to Figure 4, consider that the $i^{\text {th }}$ source undergoes a continuous time motion on a straight line constant velocity track relative to the array reference point. In this case, the time varying azimuth angle may be described as,

$$
\theta_{i}(t)=\theta_{i}\left(t_{0}\right)+\sin ^{-1}\left(\frac{v_{\theta_{i}}\left(t_{0}\right) \cdot T}{\rho_{i}\left(t_{0}\right)}\right)
$$

where $T$ is defined as the time elapsed since $t=t_{0}$ such that

$$
T=t-t_{0}
$$

and

$$
\rho_{i}(t)=\sqrt{\left(\rho_{i}\left(t_{0}\right)+v_{\rho_{i}}\left(t_{0}\right) T\right)^{2}+v_{\theta_{i}}^{2}\left(t_{0}\right) T^{2}} .
$$

Here, $\left(\rho_{i}\left(t_{0}\right), \theta_{i}\left(t_{0}\right)\right)$ and $\left(v_{\rho_{i}}, v_{\theta_{i}}\right)$ describe the components of the source location and velocity at $t=t_{0}$ respectively. Note that the time derivative of Equations 21 and 23 at $t=t_{0}$ are given by:

$$
\begin{aligned}
\left.\dot{\theta}_{i}(t)\right|_{t=t_{0}} & =\frac{v_{\theta_{i}}\left(t_{0}\right)}{\rho_{i}\left(t_{0}\right)} \\
\left.\dot{\rho}_{i}(t)\right|_{t=t_{0}} & =v_{\rho_{i}}\left(t_{0}\right)
\end{aligned}
$$

Let $T$ denote the sampling time period such that $t_{0}=(k-1) T$ and $t=k T$. The unknown source parameter vector $\underline{z}_{i}[k]$ given 
by Equation 18 can be used to define a discrete time state vector

$$
\underline{z}_{k i}=\left[\rho_{k i}, \dot{\rho}_{k i}, \theta_{k i}, \dot{\theta}_{k i}\right]^{T}
$$

which carries all the kinematic characteristics of the target. A suitable discrete time kinematic model for $t=k T$ is

$$
\underline{z}_{k i}=\mathbb{F}_{i} \underline{z}_{(k-1) i}+\underline{w}_{k i}
$$

where the state matrix $\mathbb{F}_{i} \in \mathcal{R}^{4 \times 4}$ is defined as

$$
\mathbb{F}_{i}=\left[\begin{array}{cccc}
1 & T & 0 & 0 \\
0 & 1 & 0 & 0 \\
0 & 0 & 1 & T \\
0 & 0 & 0 & 1
\end{array}\right]
$$

and the error vector $\underline{w}_{k i}$ accounts for random perturbations about the constant velocity trajectories. These perturbations can be modelled as uncorrelated acceleration noise of zero mean with covariance matrix given by

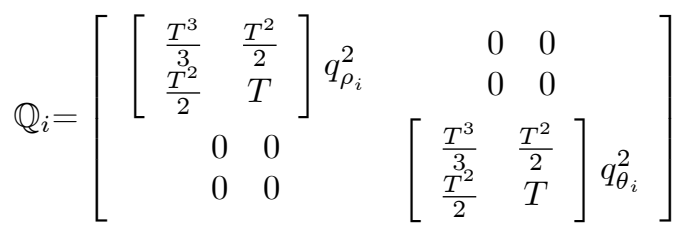

where $q_{\rho_{i}}^{2}$ and $q_{\theta_{i}}^{2}$ denote the continuous time model process noise intensity for the range-velocity and azimuth-velocity, respectively. The choice of small intensities approximates a nearly constant velocity model. Since the model is discretized, intensities have physical dimensions of length ${ }^{2} /$ time $^{3}$.

Equation 27 provides a state model describing the motion of the target which will be utilized in this paper to track its location.

\section{Multi-source Tracking Spatiotemporal-State-Space Model}

Consider now that $M$ sources are transmitting simultaneously. The received signal at the output of the TDLs is now modelled as

$$
\begin{aligned}
\underline{x}[k] & =\sum_{i=1}^{M} \underline{\mathfrak{h}}_{i}[k] \odot \underline{\mathrm{m}}_{i}[k]+\underline{n}[k] \\
& =\left[\mathbb{H}[k] \odot\left(\mathbb{M}[k] \otimes \underline{1}_{N_{s}}\right)\right] \underline{1}_{M}+\underline{n}[k]
\end{aligned}
$$

where

$$
\mathbb{M}[k] \triangleq\left[\underline{\mathrm{m}}_{1}[k], \underline{\mathrm{m}}_{2}[k], \ldots, \underline{\mathrm{m}}_{M}[k]\right] \in \mathcal{C}^{N \times M}
$$

with $\underline{\mathrm{m}}_{i}[k] \in \mathcal{C}^{N \times 1}$ describing the discretized message signal given by Equation 10 (wideband assumption), and

$$
\mathbb{H}[k] \triangleq\left[\underline{\mathfrak{h}}_{1}[k], \underline{\mathfrak{h}}_{2}[k], \cdots, \underline{\mathfrak{h}}_{M}[k]\right] \in \mathcal{C}^{N N_{s} \times M}
$$

which is time variant and has columns corresponding to the Spherical STAR manifold vectors (see Equation 19) of the $M$ sources at the $k^{\text {th }}$ time interval.

Equation 31 can be rewritten in a more useful and compact form as follows:

$$
\underline{x}[k]=\left(\mathbb{I}_{N N_{s}} \otimes \mathbb{H}[k]\right) \underline{\mathrm{m}}[k]+\underline{n}[k]
$$

where $\otimes$ denotes the Khatri-Rao row-wise product, which is the row-wise Kronecker product of two matrices. The vector $\underline{\mathrm{m}}[k] \in \mathcal{C}^{M N N_{s} \times 1}$ denotes the vectorisation of the matrix $\left(\mathbb{M}[k] \otimes \underline{1}_{N_{s}}\right)^{T}$ (see Equation 31 ) formed by stacking its columns into a single column vector. That is

$$
\underline{\mathrm{m}}[k]=\operatorname{vec}\left\{\left(\mathbb{M}[k] \otimes \underline{1}_{N_{s}}\right)^{T}\right\}
$$

Note that under the narrowband assumption Equation 34 is simplified to

$$
\begin{aligned}
\underline{x}[k] & =\sum_{i=1}^{M} \underline{\mathfrak{h}}_{i}[k] m_{i}[k]+\underline{n}[k] \\
\Rightarrow \underline{x}[k] & =\mathbb{H}[k] \underline{m}[k]+\underline{n}[k]
\end{aligned}
$$

where

$$
\underline{m}[k]=\left[m_{1}[k], m_{2}[k], \ldots, m_{M}[k]\right]^{T} .
$$

The single target discrete time kinematic model, defined in Equation 27, can be generalised to the "multi-target" case as

$$
\underline{z}_{k}=\mathbb{F} \underline{z}_{k-1}+\underline{w}_{k}
$$

where

$$
\begin{aligned}
\underline{z}_{k} & =\left[\underline{z}_{k 1}^{T}, \underline{z}_{k 2}^{T}, \cdots, \underline{z}_{k M}^{T}\right]^{T} \\
\underline{w}_{k} & =\left[\underline{w}_{k 1}^{T}, \underline{w}_{k 2}^{T}, \cdots, \underline{w}_{k M}^{T}\right]^{T} \\
\mathbb{F} & =\mathbb{I}_{M} \otimes \mathbb{F}_{i}
\end{aligned}
$$

Therefore, the covariance matrix of the discrete time state vector $\underline{z}_{k}$ defined as $\mathbb{P}_{k} \in \mathcal{R}^{4 M \times 4 M}$ is given by

$$
\mathbb{P}_{k}=\mathbb{F P}_{k-1} \mathbb{F}^{T}+\mathbb{Q}
$$

where $\mathbb{Q} \in \mathcal{R}^{4 M \times 4 M}$ is the block diagonal matrix containing all the $\mathbb{Q}_{i}$ matrices corresponding to all sources as defined in Equation 29.

\section{Summary}

To simplify the notation let us define the following parameters:

$$
\begin{aligned}
& \underline{x}_{k} \triangleq \underline{x}[k] \in \mathcal{C}^{N N_{s} \times 1} \\
& \underline{n}_{k} \triangleq \underline{n}[k] \in \mathcal{C}^{N N_{s} \times 1} \\
& \mathbb{H}_{k} \triangleq\left\{\begin{array}{l}
\stackrel{\mathrm{WB}-\mathrm{A}}{=} \mathbb{I}_{N N_{s}} \otimes \mathbb{H}[k] \in \mathcal{C}^{N N_{s} \times M N N s} \\
\stackrel{\mathrm{NB}-\mathrm{A}}{=} \mathbb{H}[k] \in \mathcal{C}^{N N_{s} \times M}
\end{array}\right. \\
& \underline{m}_{k} \triangleq\left\{\begin{array}{l}
\underline{\mathrm{m}}[k] \stackrel{\mathrm{WB}-\mathrm{A}}{=} \operatorname{vec}\left\{\left(\mathbb{M}[k] \otimes \underline{1}_{N_{s}}\right)^{T}\right\} \in \mathcal{C}^{M N N_{s} \times 1} \\
\underline{m}[k] \stackrel{\text { NB-A }}{=}\left[m_{1}[k], \ldots, m_{M}[k]\right]^{T} \in \mathcal{C}^{M \times 1}
\end{array}\right.
\end{aligned}
$$

These parameters also help to combine Equations 34 and 37 into the following equation

$$
\underline{x}_{k}=\mathbb{H}_{k} \underline{m}_{k}+\underline{n}_{k}
$$

Based on Equations 39 and 45, a state space model describing the dynamics of the motion of multiple sources and the measurement of the signals from the output of TDL at the 
array elements can be defined as follows:

$$
\left.\begin{array}{l}
\underline{z}_{k}=\mathbb{F} \underline{z}_{k-1}+\underline{w}_{k} \\
\underline{x}_{k}=\mathbb{H}_{k}\left(\underline{z}_{k}\right) \underline{m}_{k}+\underline{n}_{k}
\end{array}\right\} k>0
$$

This is the standard structure of a discrete time state space model which, however, involves the motion parameterisation described before and also the Spherical STAR manifold vectors $\underline{\mathfrak{h}}_{i}[k], i=1,2, . . M$, associated with the $M$ sources, taking into account both "narrowband" and "wideband" assumptions. With reference to Equation 46, the process noise $\underline{w}_{k} \in \mathcal{R}^{4 M \times 1}$ associated with the state space model and the measurement noise $\underline{n}_{k} \in \mathcal{C}^{N N_{s} \times 1}$ associated with the wireless channel are assumed to have zero mean and known covariance matrices $\mathbb{Q}$ and $\sigma_{n}^{2} \mathbb{I}_{N N_{s}}$ respectively. Furthermore, the initial state vector $\underline{z}_{0}$ has a mean of $\mathcal{E}\left\{\underline{z}_{0}\right\}$ and a covariance defined by the matrix $\mathbb{P}_{0}$. The first and second order statistics of $\left\{\underline{z}_{0}, \underline{w}_{k}, \underline{n}_{k}\right\}$ can be compactly restated as follows:

$$
\begin{aligned}
& \mathcal{E}\left\{\left[\begin{array}{c}
\underline{w}_{k} \\
\underline{n}_{k} \\
\underline{z}_{0} \\
1
\end{array}\right]\left[\begin{array}{c}
\underline{w}_{m} \\
\underline{n}_{m} \\
\underline{z}_{0}
\end{array}\right]^{H}\right\}=
\end{aligned}
$$

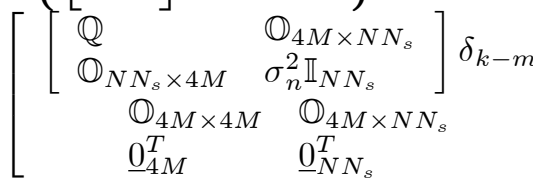$$
\begin{gathered}
\mathbb{O}_{4 M \times 4 M} \\
\mathbb{O}_{N N_{s} \times 4 M} \\
\mathbb{P}_{0} \\
\mathcal{E}\left\{\underline{z}_{0}\right\}^{H}
\end{gathered}
$$

where $\mathbb{O}_{p \times q} \in \mathcal{R}^{p \times q}$ denotes a matrix of zeros.

\section{Arrayed Extended Kalman Filter}

In the previous section the multi-target tracking problem is fully described by the discrete time state space model illustrated in Equation 46. In this section, this model will be used to adaptively estimate the state vector $\underline{z}_{k}$ based on the knowledge of the system dynamics and the received array signal vector $\underline{x}_{k}$ given by Equation 45 . In order to accommodate the nonstationary vector measurement signal and exploit the dynamical model characterizing the target's motion, a tracking approach based on Kalman filtering is used. Such an approach is convenient for handling multivariate data. In addition, it provides a significant advantage over traditional estimation techniques for the tracking problem under investigation since it is assumed that the state is a random variable to be estimated. For the tracking problem at hand, the dynamical model can be regarded as prior knowledge of the state and, therefore, can be incorporated into the estimator.

Since the measurement vector $\underline{x}_{k}$ is a nonlinear function of the state $\underline{z}_{k}$, the classic Kalman filter cannot be applied directly to estimate the state vector and hence an Extended Kalman Filtering (EKF) based approach must be developed. This is essentially a Kalman filter in which the nonlinearity is mitigated by linearizing Equation 46 at each time instant so that the traditional linear Kalman filter equations can be applied. It therefore provides a convenient way of recursively updating the target state mean and covariance matrix. Since the EKF approach developed in this section is applied to the problem of tracking targets using arrays, and Equation 46 involves Spherical STAR, it is referred to as the Arrayed
Extended Kalman Filter (Arrayed EKF). In order to apply the Arrayed EKF to this tracking problem it is assumed that the number of targets $M$ is known or has been estimated (e.g. using the MDL or AIC detection algorithms [29][30][31]) and $N N_{s}>4 M$. In addition, it is assumed that an approximation of the initial state $\underline{z}_{0}$ is provided either by using a priori guess or via some localization technique (e.g. the large aperture array localization algorithm proposed in [32]).

The Arrayed EKF needs the derivative of the Spherical STAR manifold matrix $\mathbb{H}_{k}$ with respect to the state in order the linearize the system of equations. For the $i^{t h}$ source, the gradient of the Spherical STAR manifold vector $\nabla_{\underline{z}_{i k}} \underline{\mathfrak{h}}_{i k}$ $\in \mathcal{C}^{N N_{s} \times 4}$ with respect to the state vector of the $i^{t h}$ target is

$$
\begin{aligned}
\nabla_{\underline{z}_{i k} \underline{h}_{i k}}= & \nabla_{\underline{z}_{i k}}\left(\underline{S}_{i k} \otimes \underline{1}_{N_{s}}\right) \odot\left(\underline{\mathcal{F}}_{i k} \underline{1}_{4}^{T}\right) \\
& +\left(\left(\underline{S}_{i k} \otimes \underline{1}_{N_{s}}\right) \underline{1}_{4}^{T}\right) \odot \nabla_{\underline{z}_{i k}} \underline{\mathcal{F}}_{i k}
\end{aligned}
$$

For $M$ sources, using the gradient of $\mathbb{H}\left(\underline{z}_{k}\right)$, the following matrix $\mathbb{D}_{k \mid k-1}$ can be defined as

$$
\left.\mathbb{D}_{k \mid k-1} \triangleq \nabla_{\underline{z}_{k}}\left(\mathbb{H}\left(\underline{z}_{k}\right) \underline{\hat{m}}_{k}\right)\right|_{\underline{\underline{z}}_{k \mid k-1}}
$$

Consider that the true state $\underline{z}_{k}$ is known and the objective is to estimate the message symbol $\underline{m}_{k}$ linearly from the following measurement model:

$$
\underline{x}_{k}=\mathbb{H}\left(\underline{z}_{k}\right) \underline{m}_{k}+\underline{n}_{k}
$$

where since $\underline{z}_{k}$ is known, $\mathbb{H}\left(\underline{z}_{k}\right) \in \mathcal{C}^{N N_{s} \times M}$ is known. This can be alternatively posed as estimating the matrix $\mathbb{W}$ where,

$$
\underline{\hat{m}}_{k}=\mathbb{W} \underline{x}_{k}
$$

such that

$$
\min _{\mathbb{W}} \mathcal{E}\left\{\left(\underline{m}_{k}-\mathbb{W} \underline{x}_{k}\right)^{H}\left(\underline{m}_{k}-\mathbb{W} \underline{x}_{k}\right)\right\}
$$

Using the linear model in Equation 50, the optimal solution for $\mathbb{W}$ is given by $[33]^{4}$

$$
\begin{aligned}
\mathbb{W} & =\mathbb{H}^{H}\left(\underline{z}_{k}\right)\left(\sigma_{n}^{2} \mathbb{I}_{N N_{s}}+\mathbb{H}\left(\underline{z}_{k}\right) \mathbb{H}^{H}\left(\underline{z}_{k}\right)\right)^{-1} \\
& =\frac{1}{\sigma_{n}^{2}}\left(\mathbb{I}_{M}+\frac{1}{\sigma_{n}^{2}} \mathbb{H}^{H}\left(\underline{z}_{k}\right) \mathbb{H}\left(\underline{z}_{k}\right)\right)^{-1} \mathbb{H}^{H}\left(\underline{z}_{k}\right)
\end{aligned}
$$

Since the true state is not known, the predicted estimate is used instead. Hence,

$$
\begin{gathered}
\underline{\hat{m}}_{k}=\frac{1}{\sigma_{n}^{2}}\left(\mathbb{I}_{M}+\frac{1}{\sigma_{n}^{2}} \mathbb{H}^{H}\left(\underline{\hat{z}}_{k \mid k-1}\right) \mathbb{H}\left(\underline{\hat{z}}_{k \mid k-1}\right)\right)^{-1} \\
\mathbb{H}^{H}\left(\underline{\hat{z}}_{k \mid k-1}\right) \underline{x}_{k}
\end{gathered}
$$

Based on the above discussion and equations, the Kalman filter equations can be applied directly and the proposed Arrayed EKF algorithm is summarised in Table I. Note that the difficult problem of correctly associating updated parameter estimates (ranges, directions and radial and tangential velocities) is automatically embedded in the estimation procedure - with each update associated directly with a specific spatiotemporal manifold vector (i.e. with a specific target). The predicted state vector $\widehat{\widehat{z}}_{k \mid k}$ contains all the unknowns associated with

\footnotetext{
${ }^{4}$ Note that here it is assumed in this estimator that the messages of targets are uncorrelated with one another and have unity power.
} 


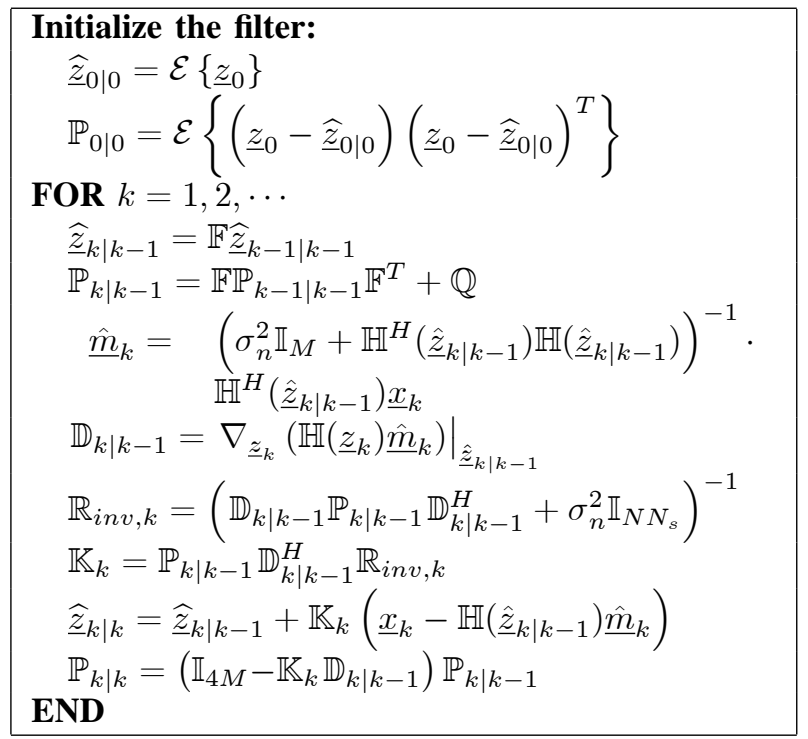

TABLE I: The proposed Arrayed EKF algorithm

the targets in a pre-specified order determined by the user from the initial locations of the targets and the spatiotemporal matrix $\mathbb{H}$. It is shown in [34] that the recursive nature of the Kalman filter forces the predicted state vector $\widehat{\underline{z}}_{k \mid k}$ to maintain this order and hence this allows the target association problem to be avoided.

Note that if the initial uncertainty is large, the initial covariance $\mathbb{P}_{0 \mid 0}$ can be set numerically to $\eta \mathbb{I}_{4 M}$ where $\eta$ is an arbitrary large number. The $\mathbb{P}_{k \mid k}$ matrix quantifies the uncertainty in the state estimates. If the nonlinearities in the system and measurement are not too severe, then the $\mathbb{P}$ matrix should give us an idea of how accurate the state estimates are. In the Arrayed EKF algorithm, the estimated message symbol can be refined by substituting the updated estimate in Equation 54 at the end of any iteration. This can be used in turn to refine the estimate of the state. Similarly, the linearization error can be reduced by reformulating the Taylor series expansion of $\mathbb{H}\left(\underline{z}_{k}\right)$ around the new estimate and recalculating the measurement update equations. These two modifications can result in an even better estimate of $\underline{z}_{k}$ and the process can be repeated as many times as desired.

\section{The Posterior Cramer-Rao Bound}

In this section the Posterior Cramer-Rao Bound (CRB) [27], also known as the Bayesian Cramer-Rao Bound, on the mean square error for the tracking problem illustrated in previous sections is derived. A recursive posterior CRB bound on the state vector $\underline{z}_{k} \in \mathcal{R}^{4 M \times 1}$ as a function of time is derived based on the discrete time nonlinear filtering bound formulated by Tichavsky et al. [35].

Let, $\underline{X}_{k} \in \mathcal{C}^{k N N s \times 1}$ and $\underline{Z}_{k} \in \mathcal{R}^{4 M(k+1) \times 1}$ denote the observations and target state vector composites respectively which comprise of the sensors measurements and the targets' motion dynamic parameters up to time $k$ such that

$$
\begin{aligned}
\underline{X}_{k} & =\left[\underline{x}_{1}^{T}, \underline{x}_{2}^{T}, \ldots, \underline{x}_{k}^{T}\right]^{T} \\
\underline{Z}_{k} & =\left[\underline{z}_{0}^{T}, \underline{z}_{1}^{T}, \ldots, \underline{z}_{k}^{T}\right]^{T}
\end{aligned}
$$

Also, let $\mathrm{p}_{\underline{X}_{k}, \underline{Z}_{k}}$ denote the joint pdf of the pair $\left(\underline{X}_{k}, \underline{Z}_{k}\right)$, $\mathcal{E}_{\underline{z}, \underline{x}}\{\cdot\}$ the expectation with respect to $\mathrm{p}_{\underline{X}_{k}, \underline{Z}_{k}}$ and the vector $\underline{\hat{Z}}_{k}$ an unbiased estimate of the state vector $\underline{Z}_{k}$ which is a function of $\underline{X}_{k}$. The Mean Square Error (MSE) matrix for the state vector composite is defined as:

$$
\operatorname{cov}\left\{\underline{Z}_{k}\right\}=\mathcal{E}_{\underline{Z}, \underline{X}}\left\{\left(\underline{\hat{Z}}_{k}-\underline{Z}_{k}\right)\left(\underline{\hat{Z}}_{k}-\underline{Z}_{k}\right)^{T}\right\}
$$

The posterior CRB is a positive definite $4 M(k+1) \times 4 M(k+1)$ matrix formulating a lower bound on the MSE matrix and is given by the inverse of the Bayesian Information Matrix $\mathbb{J}_{B}\left(\underline{Z}_{k}\right)$ as follows:

$$
\operatorname{cov}\left\{\underline{Z}_{k}\right\} \geq \mathbb{J}_{B}^{-1}\left(\underline{Z}_{k}\right)
$$

where $\mathbb{J}_{B}\left(\underline{Z}_{k}\right)$ is defined as:

$$
\mathbb{J}_{B}\left(\underline{Z}_{k}\right) \triangleq \mathcal{E}_{\underline{Z}, \underline{X}}\left\{-\nabla_{\underline{Z}} \nabla_{\underline{Z}}^{T} \ln \mathrm{p}_{\underline{X}_{k}, \underline{Z}_{k}}\right\}
$$

and can be expressed as a sum of two terms

$$
\mathbb{J}_{B}\left(\underline{Z}_{k}\right)=\mathbb{J}_{P}\left(\underline{Z}_{k}\right)+\mathbb{J}_{D}\left(\underline{Z}_{k}\right)
$$

with $\mathbb{J}_{P}\left(\underline{Z}_{k}\right)$ denoting the a priori information matrix defined by

$$
\mathbb{J}_{P}\left(\underline{Z}_{k}\right) \triangleq \mathcal{E}_{\underline{Z}}\left\{-\nabla_{\underline{Z}_{k}} \nabla_{\underline{Z}_{k}}^{T} \ln \mathrm{p}_{\underline{Z}_{k}}\right\}
$$

In the above equation $\mathcal{E}_{\underline{Z}}\{\cdot\}$ denotes the expectation with respect to $\mathrm{p}_{\underline{Z}}$ and $\mathbb{J}_{D}\left(\underline{Z}_{k}\right)$ is the contribution of the data to the information matrix, which is given by the following equation:

$$
\mathbb{J}_{D}\left(\underline{Z}_{k}\right) \triangleq \mathcal{E}_{\underline{Z}}\left\{\mathcal{E}_{\underline{X} \mid \underline{Z}}\left\{-\nabla_{\underline{Z}_{k}} \nabla_{\underline{Z}_{k}}^{T} \ln \underline{p}_{\underline{X}_{k} \mid \underline{Z}_{k}}\right\}\right\}
$$

where $\mathcal{E}_{X \mid Z}\{\cdot\}$ denotes the expectation with respect to the conditional $\mathrm{p}_{\underline{X}_{k} \mid \underline{Z}_{k}}$. Note that $\mathbb{J}_{D}\left(\underline{Z}_{k}\right)$ is the expected value of the standard Fisher Information Matrix (FIM) $\mathbb{J}\left(\underline{Z}_{k}\right)$ over the a priori distribution $\mathrm{p}_{\underline{Z}_{k}}$. That is,

$$
\mathbb{J}_{D}\left(\underline{Z}_{k}\right)=\mathcal{E}_{\underline{Z}}\left\{\mathbb{J}\left(\underline{Z}_{k}\right)\right\}
$$

Note also that $\operatorname{cov}\left\{\underline{Z}_{k}\right\}$ and $\mathbb{J}_{B}\left(\underline{Z}_{k}\right)$ are all $4 M(k+1) \times$ $4 M(k+1)$ matrices, where $M$ is the number of targets.

Let us now consider the dynamic parameters of the target at time $k$, that is $\underline{z}_{k}$. In this case then corresponding FIM $\mathbb{J}\left(\underline{z}_{k}\right)$ is the lower right $4 M \times 4 M$ block of $\mathbb{J}\left(\underline{Z}_{k}\right)$. Let us define the

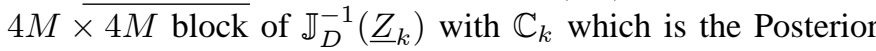
CRB for estimating $\underline{z}_{k}$ (the dynamic parameters of the target at time $k$ ). Note that $\mathbb{C}_{k}$ is the inverse of the $4 M \times 4 M$ Bayesian Information matrix $\mathbb{J}_{B, k}=\mathbb{J}_{B}\left(\underline{z}_{k}\right)$, i.e.

$$
\mathbb{C}_{k}=\mathbb{J}_{B, k}^{-1}
$$

The calculation of this matrix is generally notoriously difficult. However, assuming that the joint distribution $\mathrm{p}_{\underline{X}_{k}, \underline{Z}_{k}}$ satisfies the CRB regularity conditions [27], Tichavsky et al. [35] defined the following matrix

$$
\left[\begin{array}{ll}
\mathbb{J}_{11}, & \mathbb{J}_{12} \\
\mathbb{J}_{12}^{T}, & \mathbb{J}_{22}
\end{array}\right]
$$

with submatrices

$$
\mathbb{J}_{11}=\mathcal{E}_{\underline{z}}\left\{-\nabla_{\underline{z}_{k-1}} \nabla_{\underline{z}_{k-1}}^{T} \ln \underline{p}_{\underline{z}_{k} \mid \underline{z}_{k-1}}\right\}
$$




$$
\begin{aligned}
\mathbb{J}_{12} & =\mathcal{E}_{\underline{z}}\left\{-\nabla_{\underline{z}_{k-1}} \nabla_{\underline{z}_{k}}^{T} \ln \mathrm{p}_{\underline{z}_{k} \mid \underline{z}_{k-1}}\right\} \\
\mathbb{J}_{22} & =\mathcal{E}_{\underline{z}}\left\{-\nabla_{\underline{z}_{k}} \nabla_{\underline{z}_{k}}^{T} \ln \mathrm{p}_{\underline{z}_{k} \mid \underline{z}_{k-1}}\right\} .
\end{aligned}
$$

This provides an elegant method for calculating the $4 M \times 4 M$ information matrices $\mathbb{J}_{B, k}$ recursively as follows:

$$
\mathbb{J}_{B, k}=\underbrace{\mathbb{J}_{22}-\mathbb{J}_{12}^{T}\left(\mathbb{J}_{B, k-1}+\mathbb{J}_{11}\right)^{-1} \mathbb{J}_{12}}_{\triangleq \mathbb{J}_{P, k}(4 M \times 4 M \text { matrix })}+\mathbb{J}_{D, k}
$$

initialised using

$$
\mathbb{J}_{B, 0}=\mathcal{E}_{\underline{z}}\left\{-\nabla_{\underline{z}_{0}} \nabla_{\underline{z}_{0}}^{T} \ln \mathrm{p}_{\underline{z}_{0}}\right\}
$$

where

$$
\begin{aligned}
\mathbb{J}_{D, k} & =\mathcal{E}_{\underline{x}, \underline{z}}\left\{-\nabla_{\underline{z}_{k}} \nabla_{\underline{z}_{k}}^{T} \ln \mathrm{p}_{\left.\underline{x}_{k} \mid \underline{z}_{k}\right\}}\right. \\
& =\mathcal{E}_{\underline{z}}\left\{\mathcal{E}_{\underline{x} \mid \underline{z}}\left\{-\nabla_{\underline{z}_{k}} \nabla_{\underline{z}_{k}}^{T} \ln \mathrm{p}_{\underline{x}_{k} \mid \underline{z}_{k}}\right\}\right\} \\
& =\mathcal{E}_{\underline{z}}\left\{\mathbb{J}_{k}\right\} .
\end{aligned}
$$

Note that the matrix $\mathbb{J}_{k}$ in Equation 71 is the standard FIM for estimating the state vector $\underline{z}_{k}$ based on the observation $\underline{x}_{k}$. The recursion involves computations with matrices of dimension $4 M \times 4 M$.

Following the previous discussion, the state space model described in Equations 46 and 47 has a linear additive white Gaussian noise process model and a nonlinear measurement model. The Bayesian Information Matrix $\mathbb{J}_{B, k}$ can be computed recursively using Equation 69 by evaluating the matrices defined in Equations 66 to 68 and 71. A common term in the definition of each of the matrices $\mathbb{J}_{22}, \mathbb{J}_{11}, \mathbb{J}_{12}$ is $-\ln \mathrm{p}_{\underline{z}_{k} \mid \underline{z}_{k-1}}$ which can be simplified using the Gaussian structure of the process state model in the tracking problem in hand as follows:

$$
-\ln \mathrm{p}_{\underline{z}_{k} \mid \underline{z}_{k-1}}=\frac{1}{2}\left(\underline{z}_{k}-\mathbb{F} \underline{z}_{k-1}\right)^{T} \mathbb{Q}^{-1}\left(\underline{z}_{k}-\mathbb{F} \underline{z}_{k-1}\right)+c_{0}
$$

where $c_{0}$ denotes a constant independent of $\underline{z}_{k}, \underline{z}_{k-1}$. As a result, the $4 M \times 4 M$ recursion of the Bayesian Information matrix (see Equation 69) has the form

$$
\mathbb{J}_{B, k}=\underbrace{\triangleq \mathbb{J}_{22}}_{\mathbb{\mathbb { Q }}_{P, k}^{-1}}-\overbrace{\mathbb{Q}^{-1} \mathbb{F}}^{\triangleq-J_{12}^{T}}(\mathbb{J}_{B, k-1}+\overbrace{\mathbb{F}^{T} \mathbb{Q}^{-1} \mathbb{F}}^{\triangleq \mathbb{J}_{11}})^{-1} \overbrace{\mathbb{F}^{T} \mathbb{Q}^{-1}}^{\triangleq}+\mathbb{J}_{D, k}
$$

By applying the familiar matrix inversion lemma (refer to [36]), the term in the previous equation which represents the matrix $\mathbb{J}_{P, k-1}$ can be written in a more compact way as

$$
\begin{aligned}
\mathbb{J}_{P, k} & =\left(\mathbb{Q}+\mathbb{F} \mathbb{J}_{B, k-1}^{-1} \mathbb{F}^{T}\right)^{-1} \\
& =\left(\mathbb{Q}+\mathbb{F} \mathbb{C}_{k-1} \mathbb{F}^{T}\right)^{-1}
\end{aligned}
$$

In order to evaluate the contribution of the data to the information matrix represented by the matrix $\mathbb{J}_{D, k}$, a careful analysis of the observation model is needed. From Equation 71 , the matrix $\mathbb{J}_{D, k}$ is obtained from the expected value of the standard Fisher Information matrix $\mathbb{J}_{k}$ of the array observation data model. The array output is a temporally white random vector with zero mean and covariance matrix:

$$
\mathbb{R}_{x_{k} x_{k}}=\mathbb{H}_{k} \mathbb{R}_{m_{k} m_{k}} \mathbb{H}_{k}^{H}+\sigma_{n}^{2} \mathbb{I}_{N N_{s}}
$$

where $\mathbb{R}_{m_{k} m_{k}} \in \mathcal{C}^{M \times M}$ denotes the signal covariance matrix. The standard FIM for estimating the composite state vector $\underline{z}_{k}$ for all targets can be seen as a direct extension to the work in [37] and has the following form:

$$
\begin{aligned}
\mathbb{J}_{k}= & \frac{2}{\sigma_{n}^{2}} \operatorname{Re}\left(\mathbb{D}_{k}^{H} \mathbb{P}_{\mathbb{H}_{k}}^{\perp} \mathbb{D}_{k}\right) \odot \\
& \left(\mathbb{R}_{m_{k} m_{k}} \mathbb{H}_{k}^{H} \mathbb{R}_{x_{k} x_{k}}^{-1} \mathbb{H}_{k} \mathbb{R}_{m_{k} m_{k}} \otimes\left(\underline{1}_{4} \underline{1}_{4}^{T}\right)\right)^{T}
\end{aligned}
$$

where $\mathbb{D}_{k}$ is given by

$$
\mathbb{D}_{k}=\nabla_{\underline{z}_{k}}\left(\mathbb{H}_{k}\right)
$$

and the matrix $\mathbb{P}_{\mathbb{H}_{k}}^{\frac{1}{2}}$ denotes the projection operator on the complement subspace spanned by the columns of $\mathbb{H}_{k}$ and defined as $\mathbb{P}_{\mathbb{H}_{k}}^{\perp}=\mathbb{I}_{N N_{s}}-\mathbb{H}_{k}\left(\mathbb{H}_{k}^{H} \mathbb{H}_{k}\right)^{-1} \mathbb{H}_{k}^{H}$. For simplicity, signals from different targets are assumed to be uncorrelated and, therefore, the signal covariance matrix $\mathbb{R}_{m_{k} m_{k}}$ is reduced to the unity matrix $\mathbb{I}_{M}$. Consequently, Equation 76 becomes as follows:

$$
\mathbb{J}_{k}=\frac{2}{\sigma_{n}^{2}} \operatorname{Re}\left(\mathbb{D}_{k}^{H} \mathbb{P}_{\mathbb{H}_{k}}^{\perp} \mathbb{D}_{k}\right) \odot\left(\mathbb{H}_{k}^{H} \mathbb{R}_{x_{k} x_{k}}^{-1} \mathbb{H}_{k} \otimes\left(\underline{1}_{4} \underline{1}_{4}^{T}\right)\right)^{T}
$$

As a result, the total Bayesian Information Matrix recursion can be given by:

$$
\begin{aligned}
& \mathbb{J}_{B, k}=\underbrace{\left(\mathbb{Q}+\mathbb{F} \mathbb{J}_{B, k-1}^{-1} \mathbb{F}^{T}\right)^{-1}}_{\triangleq \mathbb{J}_{P, k}} \\
& +\underbrace{\mathcal{E}_{\underline{z}_{k}}\left\{\frac{2}{\sigma_{n}^{2}} \operatorname{Re}\left(\mathbb{D}_{k}^{H} \mathbb{P}_{\mathbb{H}_{k}}^{\perp} \mathbb{D}_{k}\right) \odot\left(\mathbb{H}_{k}^{H} \mathbb{R}_{x_{k} x_{k}}^{-1} \mathbb{H}_{k} \otimes\left(\underline{1}_{4} \underline{1}_{4}^{T}\right)\right)^{T}\right\}}_{\triangleq \mathbb{J}_{D, k}}
\end{aligned}
$$

The expectation in the above equation is generally evaluated using Monte Carlo techniques.

For a single target $M=1$, the total Bayesian Information Matrix recursion is greatly reduced to:

$$
\begin{aligned}
\mathbb{J}_{B, k}= & \underbrace{\left(\mathbb{Q}+\mathbb{F} \mathbb{J}_{B, k-1}^{-1} \mathbb{F}^{T}\right)^{-1}}_{\triangleq \mathbb{J}_{P, k}} \\
& \underbrace{\mathcal{E}_{\underline{z}_{k}}\{\frac{2}{\sigma_{n}^{2}} \cdot \underbrace{\frac{c_{k}}{c_{k}+\sigma_{n}^{2}}}_{\triangleq \epsilon_{k}} \operatorname{Re}\left(\mathbb{D}_{k} \mathbb{P}_{\mathbb{H}_{k}}^{\perp} \mathbb{D}_{k}\right)\}}_{\triangleq \mathbb{J}_{D, k}}
\end{aligned}
$$

where the term $c_{k}$ is given by

$$
c_{k}=N_{s} \underline{1}_{N}^{T}\left(\rho_{k}^{2} \underline{1}_{N} \oslash \underline{d}_{k}^{2}\right)
$$

Note that according to the above equation, $c_{k}$ is a positive number greater than 1 and it monotonically increases with the length of the TDL $\left(N_{s}\right)$ and the number of array elements $(N)$. Note also that $\epsilon_{k}$ in Equation 80 ranges between 0 and 1 and $\epsilon_{k} \rightarrow 1$ when $N_{s} \rightarrow \infty$ or $\sigma_{n}^{2} \rightarrow 0$.

Now, some useful properties of the Bayesian Information Matrix are established. Specifically, the effect of increasing the 
number of sensor nodes $(N)$ and the length of the TDL $\left(N_{s}\right)$ on the Bayesian Information Matrix recursion is investigated. In order to do that, some auxiliary results are firstly established.

Lemma 1: Consider the matrices $\mathbb{Q}, \mathbb{F}$ defined in Section II-B and the a priori information matrix $\mathbb{J}_{P, k}$ given by Equation 74. Let $\mathbb{J}_{B, k}(N)$ be the Bayesian Information matrix using $N$ sensors at time $k$. If

$$
\mathbb{J}_{B, k-1}(N+1) \geq \mathbb{J}_{B, k-1}(N)
$$

then the a priori information recursion at any time $k-1$ increases monotonically with increasing $N$. That is the matrix $\mathbb{J}_{P, k}$ satisfies the following order:

$$
\mathbb{J}_{P, k}(N+1) \geq \mathbb{J}_{P, k}(N)
$$

Similarly, let $\mathbb{J}_{B, k}\left(N_{s}\right)$ be the Bayesian Information Matrix using a TDL of length $N_{s}$. If

$$
\mathbb{J}_{B, k-1}\left(N_{s}+1\right) \geq \mathbb{J}_{B, k-1}\left(N_{s}\right)
$$

then

$$
\mathbb{J}_{P, k}\left(N_{s}+1\right) \geq \mathbb{J}_{P, k}\left(N_{s}\right)
$$

Proof: The proof of this lemma uses some of the matrix properties included in [36]. Since $\mathbb{J}_{B, k-1}(N+1)$ and $\mathbb{J}_{B, k-1}(N)$ are Hermitian positive definite matrices for all time $k$ and if $\mathbb{J}_{B, k-1}(N+1) \geq \mathbb{J}_{B, k-1}(N)$, then:

$$
\mathbb{J}_{B, k-1}^{-1}(N+1) \leq \mathbb{J}_{B, k-1}^{-1}(N)
$$

The previous inequality is preserved by pre and post multiply by $\mathbb{F}$ and $\mathbb{F}^{T}$,

$$
\mathbb{F} \coprod_{B, k-1}^{-1}(N+1) \mathbb{F}^{T} \leq \mathbb{F} \rrbracket_{B, k-1}^{-1}(N) \mathbb{F}^{T}
$$

Therefore, since the matrix $\mathbb{Q}$ is positive definite, the following order is satisfied:

$$
\mathbb{F} \mathbb{J}_{B, k-1}^{-1}(N+1) \mathbb{F}^{T}+\mathbb{Q} \leq \mathbb{F} \mathbb{J}_{B, k-1}^{-1}(N) \mathbb{F}^{T}+\mathbb{Q}
$$

By inverting both sides of the inequality, the following result is obtained :

$$
\begin{aligned}
\left(\mathbb{F} \mathbb{J}_{B, k-1}^{-1}(N+1) \mathbb{F}^{T}+\mathbb{Q}\right)^{-1} & \geq\left(\mathbb{F} \coprod_{B, k}^{-1}(N) \mathbb{F}^{T}+\mathbb{Q}\right)^{-1} \\
\Rightarrow \mathbb{J}_{P, k}(N+1) & \geq \mathbb{J}_{P, k}(N)
\end{aligned}
$$

This concludes the proof of the first part. The second part can be proved in a similar way.

Lemma 2: Consider the matrix $\mathbb{J}_{D, k}$ defined in Equation 79. $\mathbb{J}_{D, k}$ at any time $k$ increases monotonically with increasing the number of sensors $(N)$ or increasing the TDL length $\left(N_{s}\right)$. That is the matrix $\mathbb{J}_{D, k}$ satisfies the following order:

$$
\begin{aligned}
\mathbb{J}_{D, k}(N+1) & \geq \mathbb{J}_{D, k}(N) \\
\mathbb{J}_{D, k}\left(N_{s}+1\right) & \geq \mathbb{J}_{D, k}\left(N_{s}\right)
\end{aligned}
$$

for each time instant $k$ where $\mathbb{J}_{D, k}(N)$ denotes the $\mathbb{J}_{D, k}$ matrix using $N$ sensors and $\mathbb{J}_{D, k}\left(N_{s}\right)$ is the $\mathbb{J}_{D, k}$ matrix using a TDL of length $N_{s}$.

Theorem 3: The Bayesian Information Matrix recursion at any time $k$ increases monotonically with increasing the number of array sensors $(N)$ or the TDL length $\left(N_{s}\right)$. That is the matrix $\mathbb{J}_{B, k}$ satisfies the following order relations:

$$
\begin{gathered}
\mathbb{J}_{B, k}(N+1) \geq \mathbb{J}_{B, k}(N) \\
\mathbb{J}_{B, k}\left(N_{s}+1\right) \geq \mathbb{J}_{B, k}\left(N_{s}\right)
\end{gathered}
$$

Proof: From Equation 79, in order to prove this theorem, it is sufficient to prove that the following two relations hold:

1. $\mathbb{J}_{D, k}\left(N_{d}+1\right) \geq \mathbb{J}_{D, k}\left(N_{d}\right)$

2. $\mathbb{J}_{P, k}\left(N_{d}+1\right) \geq \mathbb{J}_{P, k}\left(N_{d}\right)$

where $N_{d}$ denotes the number of sensors or the length of the TDL line, i.e., $N_{d}=N$ or $N_{d}=N_{s}$.

The first inequality directly follows from Lemma 2. To prove the second inequality, Lemma 1 is used such that the problem is reduced to proving the following inequality:

$$
\mathbb{J}_{B, k-1}\left(N_{d}+1\right) \geq \mathbb{J}_{B, k-1}\left(N_{d}\right)
$$

However, the Bayesian Information Matrix recursion is initialised using

$$
\mathbb{J}_{B, 0}=\mathcal{E}_{\underline{z}}\left\{-\nabla_{\underline{z}_{0}} \nabla_{\underline{z}_{0}}^{T} \ln p\left(\underline{z}_{0}\right)\right\}
$$

which is independent of $N_{d}$. Therefore, since,

$$
\mathbb{J}_{D, 1}\left(N_{d}+1\right) \geq \mathbb{J}_{D, 1}\left(N_{d}\right)
$$

the Bayesian Information Matrix recursion $\mathbb{J}_{B, 1}$ satisfies the following order:

$$
\mathbb{J}_{B, 1}\left(N_{d}+1\right) \geq \mathbb{J}_{B, 1}\left(N_{d}\right)
$$

Given $\mathbb{J}_{B, 1}$, from the second condition, it can be easily deduced that:

$$
\mathbb{J}_{B, k-1}\left(N_{d}+1\right) \geq \mathbb{J}_{B, k-1}\left(N_{d}\right)
$$

which in turn guarantees the second relation and completes the proof.

The above theorem (Theorem 3) highlights the positive effect of increasing the number of array sensors and the TDL length on the performance of the tracking algorithm. Thus by utilizing more array elements and/or a longer TDL length for the "difficult" paths/intervals of the tracking process, the estimation can be improved even further. This is of particular importance in sensor networks where the availability of redundant nodes can be utilized to increase the number of nodes forming an array system in difficult tracking situations, e.g. crossing situations.

\section{Performance Evaluation}

The simulation environment is described as follows. The array used for tracking is defined as

$$
\mathbf{r}=\left[\begin{array}{ccccccc}
0 & 50 & 30 & -30 & 30 & 10 & -50 \\
0 & 0 & 45 & -50 & -62 & 75 & 29 \\
0 & 0 & 0 & 0 & 0 & 0 & 0
\end{array}\right]
$$

measured in meters. As can be seen, the array does not possess any special geometrical structure. Two narrowband sources of equal power are tracked using the array. The signals of the sources are simulated as independent identically distributed Gaussian processes with a unity variance and the carrier frequency $F_{c}$ is assumed to be $2.4 \mathrm{GHz}$. The targets are 


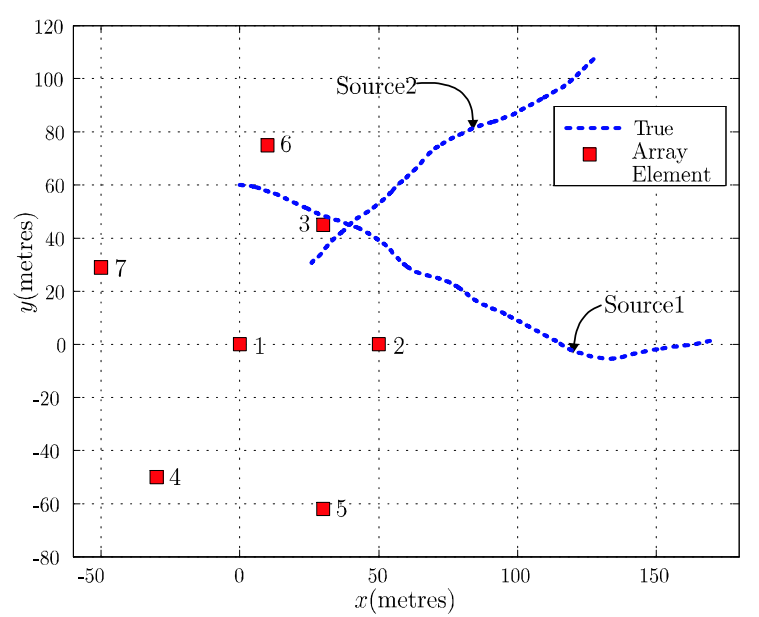

Fig. 5: Position Tracking: True trajectories of two sources to be tracked using the $N=7$ element array in Equation 86 .

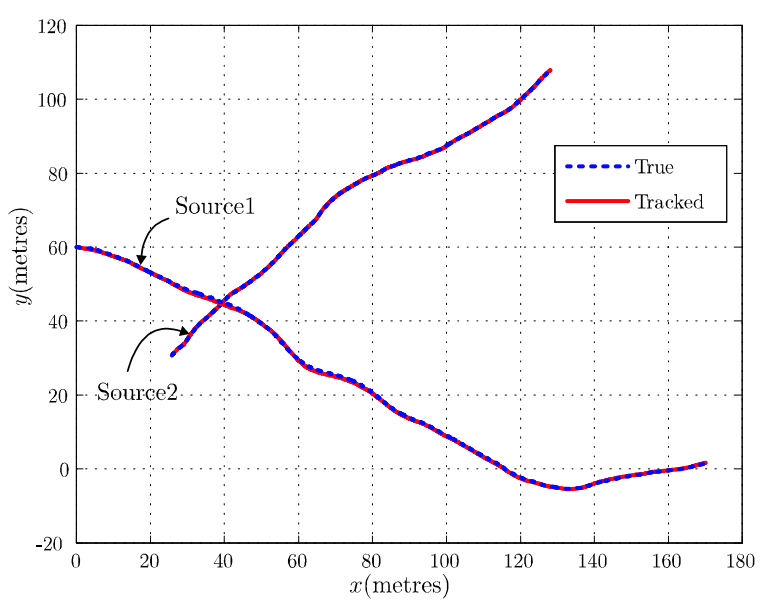

Fig. 6: Position Tracking: True trajectories and tracked positions using the proposed algorithm for two sources at $\mathrm{SNR}_{i n}=$ $0 \mathrm{~dB}$ for the environment of Figure 5 with $N_{s}=10$.

moving in the same plane as the sensor array. The array elements and the trajectories of the sources in the $x-y$ plane are shown (blue dotted lines) in Figure 5. It is important to note that Figure 5 shows targets crossing in space. However, by studying the trajectories of these targets as a function of range and direction over time, as shown in Figures 7 and 8, it can be seen that that this crossing doesn't occur at the same time. In particular, in Figure 7 it is shown that the targets have the same range at times $0.9 \mathrm{~s}$ and $4.5 \mathrm{~s}$ but don't have the same direction at these times relative to the array reference point. Furthermore, in Figure 8 it is shown that they have the same direction at time $1.5 \mathrm{~s}$ but not the same range. In this paper, we also consider the crossing situation where targets cross in space at the same time.

Tracking is assumed to be performed over an interval of $5 \mathrm{~s}$ with $T=1 \mathrm{~ms}$. Hence, there are a total of 5000 snapshots of data are available at the end of the tracking interval. When

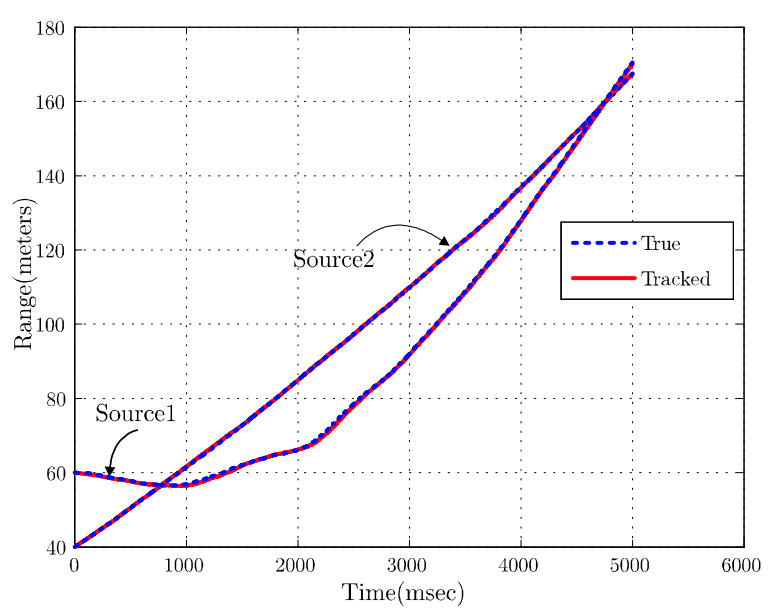

Fig. 7: Range Tracking: True and tracked ranges using the proposed algorithm for two sources at $\mathrm{SNR}_{i n}=0 \mathrm{~dB}$ for the environment of Figure 5 with $N_{s}=10$.

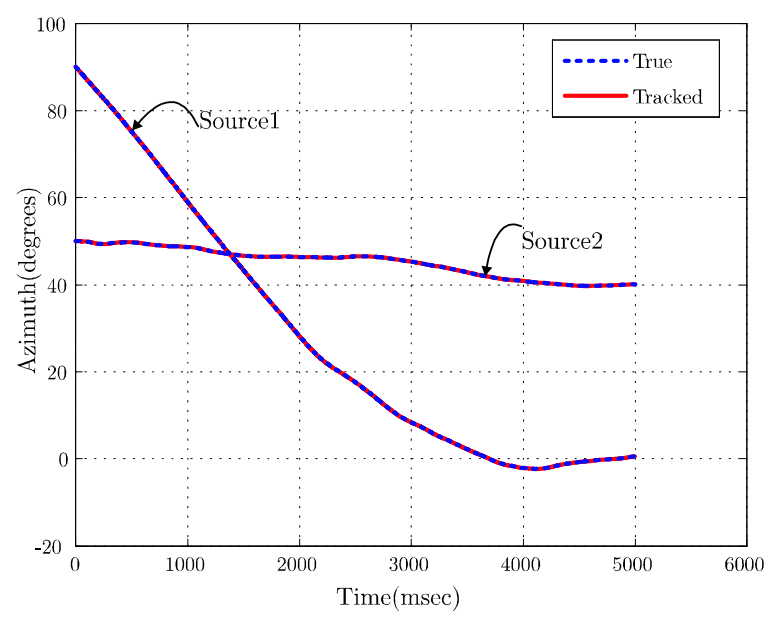

Fig. 8: Azimuth Tracking: True and tracked azimuth angles using the proposed algorithm for two sources at $\mathrm{SNR}_{i n}=0$ $\mathrm{dB}$ for the environment of Figure 5 with $N_{s}=10$.

applying the tracking algorithm, the initial locations of the targets are assumed to be known to within 1 meter (e.g. estimated using any high resolution localization approach as in [32]) with initial covariance matrix $\mathbb{P}_{0 \mid 0}=\mathbb{I}$. The selected level of the range process noise is $q_{\rho_{i}}^{2}=30 \mathrm{~m}^{2} / \mathrm{sec}^{3}$ and the direction (angle) process noise is $q_{\theta_{i}}^{2}=0.035 \mathrm{rad}^{2} / \mathrm{sec}^{3}$, where $i=1,2$, Note that it is impractical to assume full knowledge of the true process noise statistics (covariance matrix). However, any "uncertainty" has no significant effect in the tracking performance.

\section{A. The effect of $S N R_{\text {in }}$}

For the purposes of simulation, the length of TDL for each element is assumed to be $10\left(N_{s}=10\right)$. The array data is simulated for $0 \mathrm{~dB} \mathrm{SNR}_{i n}$. 


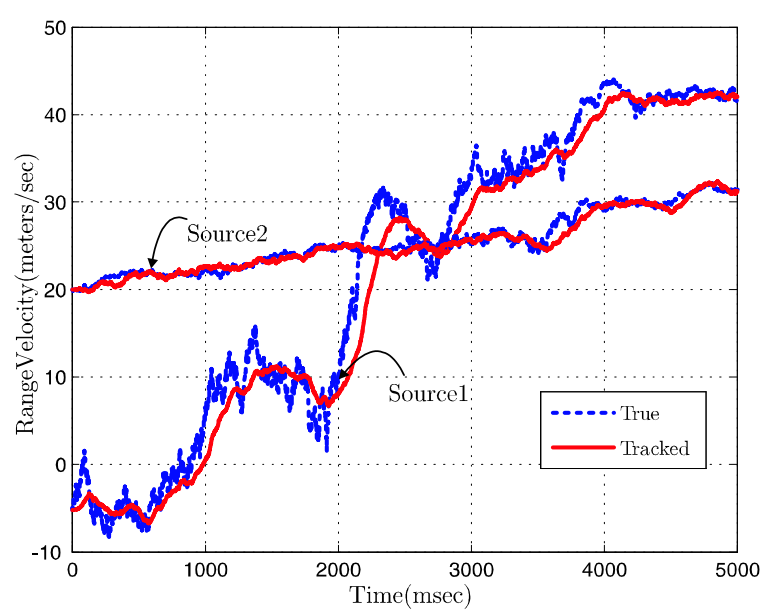

Fig. 9: Radial Velocity Tracking: True and tracked radial velocity using the proposed algorithm for two sources at $\mathrm{SNR}_{i n}=0 \mathrm{~dB}$ for the environment of Figure 5 with $N_{s}=10$.

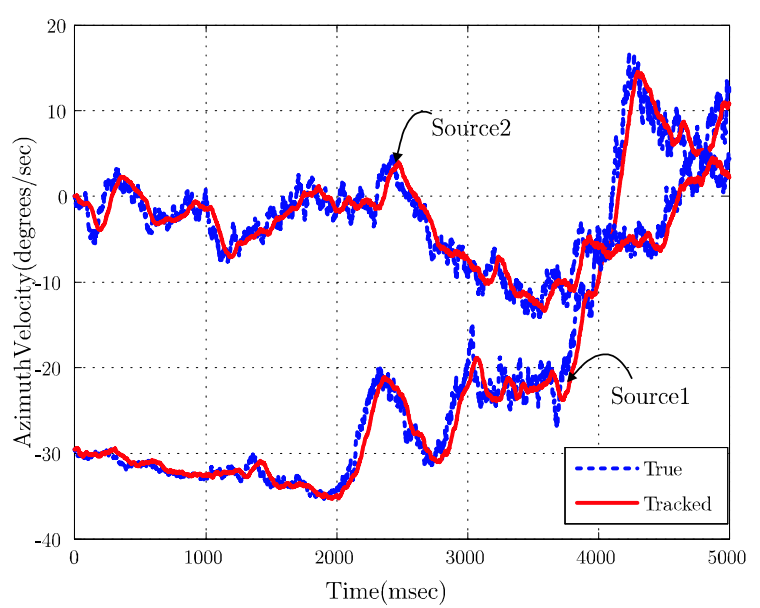

Fig. 10: Tangential Velocity Tracking: True and tracked tangential velocity using the proposed algorithm for two sources at $\mathrm{SNR}_{i n}=0 \mathrm{~dB}$ for the environment of Figure 5 with $N_{s}=10$.

Figure 5 provides a plot of the two target trajectories. Figures 6 to 10 provide a plot of the true target motion parameters in dotted blue lines superimposed by red solid lines representing the corresponding estimated parameters in a typical sample run.

From Figures 6, 7 and 8, it is clear that the proposed tracking algorithm accurately estimates the location parameters of the 2 targets for $\mathrm{SNR}_{i n}=0 \mathrm{~dB}$. In addition, the radial and orthoradial velocity for targets are estimated with a relatively good performance at low $\mathrm{SNR}_{i n}$ values (see Figures 9 and 10).

In order to demonstrate computation and behaviour of the Posterior CRB bound, the effect of the $\mathrm{SNR}_{i n}$ on the Posterior CRB have been examined by using Equation 79. The expectation-operator in the posterior part of the information

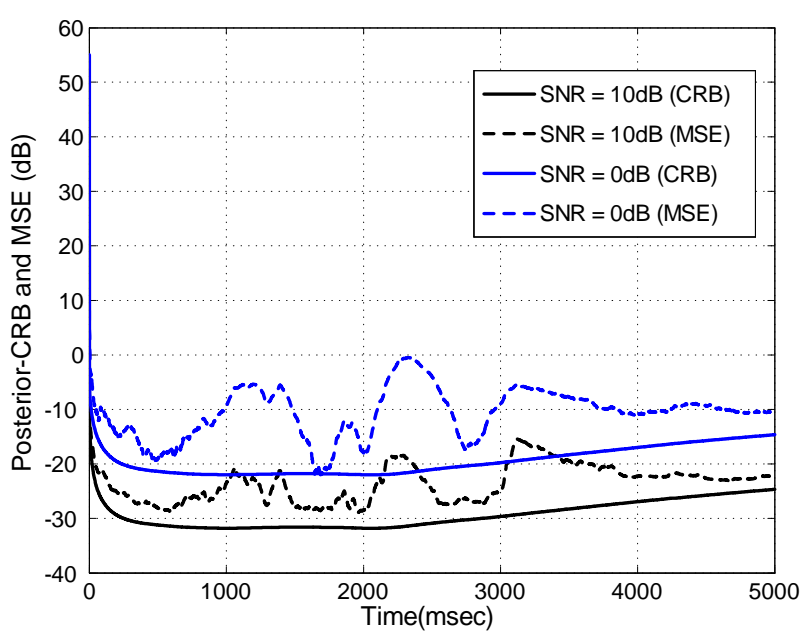

Fig. 11: Range PCRB and MSE: Comparison between the Posterior CRB and the MSE of the estimated range of the 1 st moving source associated with Figure 7.

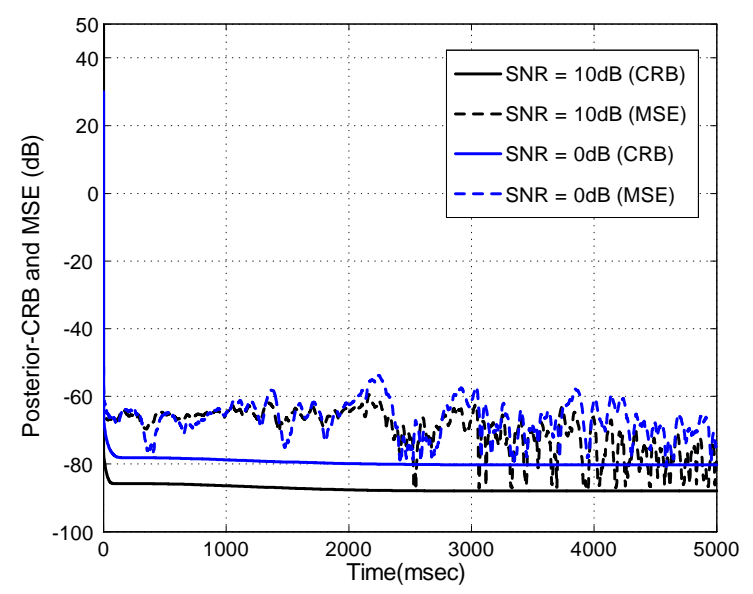

Fig. 12: Azimuth PCRB and MSE: Comparison between the Posterior CRB and the MSE of the estimated direction of the 1 st moving source associated with Figure 8.

matrix in Equation 79 was numerically computed using 50000 Monte Carlo simulation runs. Figures 11 and 12 show the results of the range-PCRB and azimuth-PCRB, respectively, for tracking the range and the angle of one of the two targets (Target 1 ) for $\mathrm{SNR}_{i n}=0 \mathrm{~dB}$ (blue cont.-line) and $\mathrm{SNR}_{\text {in }}=10$ $\mathrm{dB}$ (black cont.-line).

$\pi$ As expected, both figures show that the Posterior CRB decreases monotonically with increasing the value of $\mathrm{SNR}_{i n}$. For comparison, Figures 11 and 12 also show the mean square error (MSE) of the Arrayed EKF tracking algorithm estimates, for $\mathrm{SNR}_{i n}=0 \mathrm{~dB}$ (blue dotted-line) and $\mathrm{SNR}_{\text {in }}=10$ $\mathrm{dB}$ (black dotted-line). In particular, the tracking algorithm estimates are utilized for the numerical computation of the mean-square error matrix given by:

$$
\hat{\mathbb{P}}_{k \mid k}=\frac{1}{L} \sum_{l=1}^{L}\left(\underline{\hat{z}}_{k \mid k}(l)-\underline{z}_{k}\right)\left(\underline{\hat{z}}_{k \mid k}(l)-\underline{z}_{k}\right)^{T}
$$


where the estimates were averaged ${ }^{5}$ over $L=50000$ Monte Carlo trials. It is interesting to note that the MSE of the proposed Arrayed EKF tracking algorithm is very low and come very close to achieving the bound at some time instants.

\section{B. The Effect of Increasing the TDL Length}

In this section the effect of increasing the dimensionality of the spatiotemporal manifold vector is examined. The dimension of this manifold vector can be increased by increasing either the length of the TDL $\left(N_{s}\right)$ in each array element or the number of array elements $(N)$. In this section, the effect of increasing $N_{s}$ on the tracking performance is chosen for investigation. Note that increasing the array size $(N)$ at fixed $\mathrm{SNR}_{i n}$ will have the same effect. Two different sets of array data are simulated at $\mathrm{SNR}_{i n}$ equal to $-10 \mathrm{~dB}$ for two TDL lengths: $N_{s}=10$ and $N_{s}=20$. The aim is to track the trajectories of two targets shown in Figure 5 using same array defined in Equation 86. Figure 13 provides a sample run estimate of the target position and the corresponding real position for each of the two TDL lengths. From Figure 13(a) it is clear that the tracking algorithm exhibits poor performance in tracking the motion parameters of the targets' trajectories when $N_{s}=10$. On the other hand, the estimation accuracy is significantly improved by increasing $N_{s}$ to 20 as observed in Figure 13(b). These results indicate that the tracking algorithm improves significantly as the length of the TDL increases.

\section{Crossing Situations}

All the trajectories of the targets displayed in the previous figures represent situations where targets may have the same range or direction at any instant of time but not both. In other words, the targets do not cross in space at the same time instant. In this section crossing situations are considered where targets cross in space at the same time. In the scenario under investigation, two targets cross in space at $2.5 \mathrm{~s}$ at different velocities. The following table shows the main parameters of this scenario.

\begin{tabular}{||l|l||l|l||}
\hline \hline Parameter & Value & Parameter & Value \\
\hline \hline SNR $_{i n}(\mathrm{~dB})$ & 10 & Number of Targets $(M)$ & 2 \\
TDL length $\left(N_{s}\right)$ & 10 & Number of Sensors $(N)$ & 7 \\
\hline \hline
\end{tabular}

Figure 14, 15 and 16 illustrate one of the distinguishing features of the proposed Arrayed EKF model; since velocities (radial and tangential) are incorporated in the observation model, targets crossing can be easily resolved if velocities are sufficiently different. However, if the velocities of the crossing targets are within the standard deviation of the measurement error then the corresponding spatiotemporal manifold vectors of the crossing targets tend to become collinear - and this degrades the tracking performance as the two crossing targets appear in the array as one. To alleviate this problem, the number of array elements is increased which, consequently,

\footnotetext{
${ }^{5}$ Results with 50 and 1000 Monte-Carlo trials have shown that the MSE occasionally falls below the PCBR. However, using a large number of trials, the MSE curves remains above the PCRB - as theoretically expected.
}

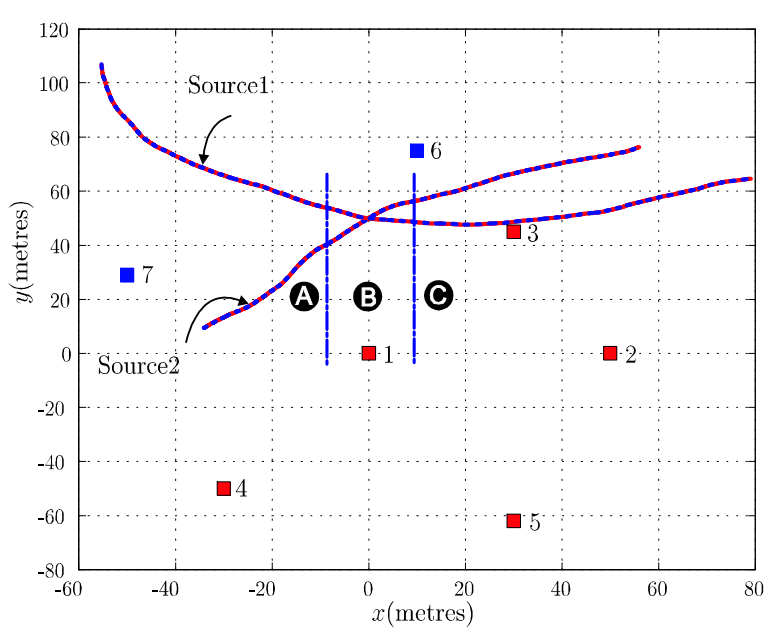

Fig. 14: Position Tracking for the Crossing Situation: True trajectories and tracked positions using the proposed algorithm for two crossing sources at $\mathrm{SNR}_{i n}=10 \mathrm{~dB}$ with $N_{s}=10$. An array of 5 sensors is used in interval A, increasing to 7 in interval $\mathrm{B}$ and reducing again to 5 in interval $\mathrm{C}$. The red squares represent the initial 5 sensor array. The blue squares represent the two additional sensors used to form the 7 sensor array in the interval B.

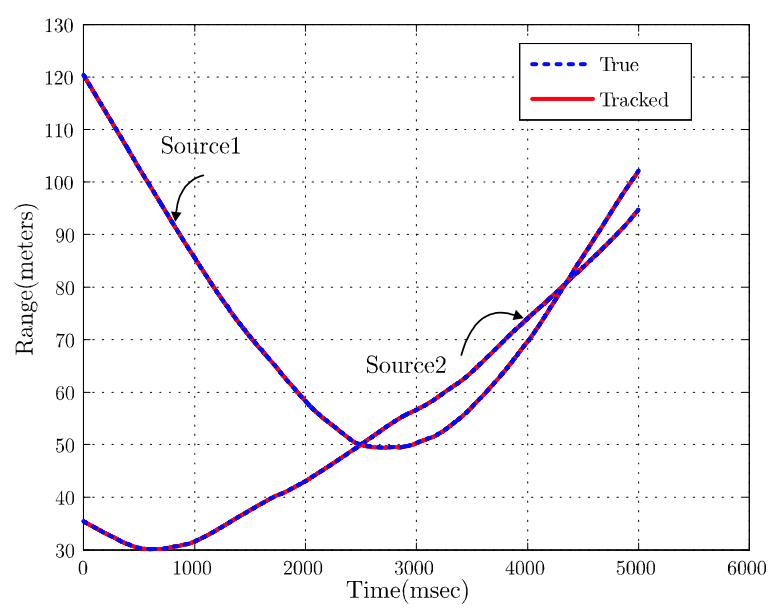

Fig. 15: Range Tracking for the Crossing Situation: True and tracked ranges using the proposed algorithm for two crossing sources for the environment of Figure 14.

increases the degrees-of-freedom and thus increases the angle between the new (longer) spatiotemporal manifold vectors around the crossing point. That is, as targets get closer to each other, i.e. when a crossing situation is predicted, the number of array elements is increased to alleviate any degradation in the tracking performance.

In practice, the controller of an array can wake up nodes in its communication range when detecting a possible crossing situation. Figure 14 shows the scenario under investigation where only the first 5 elements of the array defined in Equation 


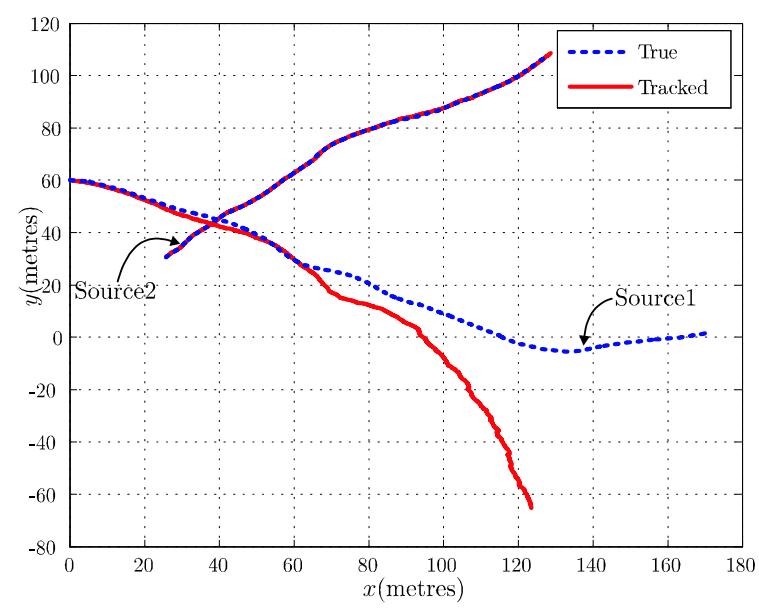

(a)

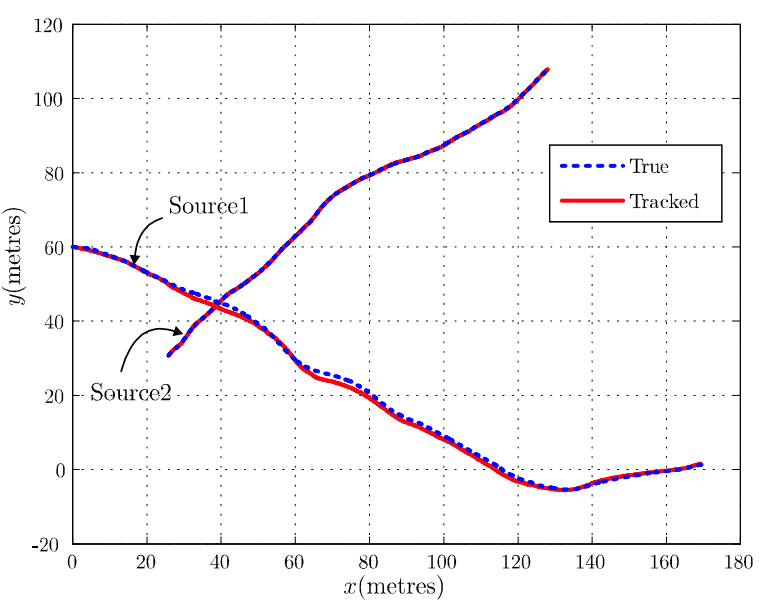

(b)

Fig. 13: Position Tracking with Variable TDL Length: True trajectories and tracked positions using the proposed algorithm for two sources at $\mathrm{SNR}_{i n}=-10 \mathrm{~dB}$ for the environment of Figure 5 with: (a) $N_{s}=10$ (b) $N_{s}=20$.

86 are initially used to track the two targets (region $\mathrm{A}$ in Figure 14). This is increased to using all 7 elements after 2 seconds as targets begin to become closer to each other (region B). After 3 seconds, targets are sufficiently far from each other and the algorithm reverts back to using the initial 5 element array (region $\mathrm{C}$ ). Note that in each region, all parameters apart from the number of array sensors remains fixed. Figures 15 and 16 show the tracking of range and direction, respectively of the proposed approach. Furthermore, Figure 17 shows the computational complexity of the proposed algorithm with $N$ changing from 5 to 7 to 5 array elements. For comparison, the same figure also shows the computational complexity of the proposed algorithm when $N$ is fixed at 7 . The total computational saving over the entire tracking interval in this scenario is over $20 \%$. Figure 18 shows the RMSE for tracking the same trajectory using $N=5, N=7$ and also $N=5$ to 7 to 5 . The total mean square error increased from $0.0012 \mathrm{~m}$ using 7 sensors to $0.0014 \mathrm{~m}$ using the "adaptive" $N$ sensors.

\section{CONCLUSIONS}

In this paper the problem of tracking multiple moving targets was tackled using large aperture arrays. Firstly, a novel spherical-spatiotemporal-state-space model was presented incorporating time varying functions of the ranges, directions and Doppler effects of the targets in conjunction with the array geometry. Then, this was integrated with the concept of the Extended Kalman Filter (EKF) to propose a novel spacetime self-adaptive approach to track multiple moving targets. In addition, based on the Posterior Cramer-Rao Bound (Posterior $\mathrm{CRB}$ ), a recursive lower bound on the error covariance matrix of the tracking problem was calculated. A theorem that explains the effects of various parameters on the Posterior CRB was developed. In particular, the impact of increasing the number of sensors and the length of the tapped delay line (TDL) on the Posterior CRB was investigated. It was

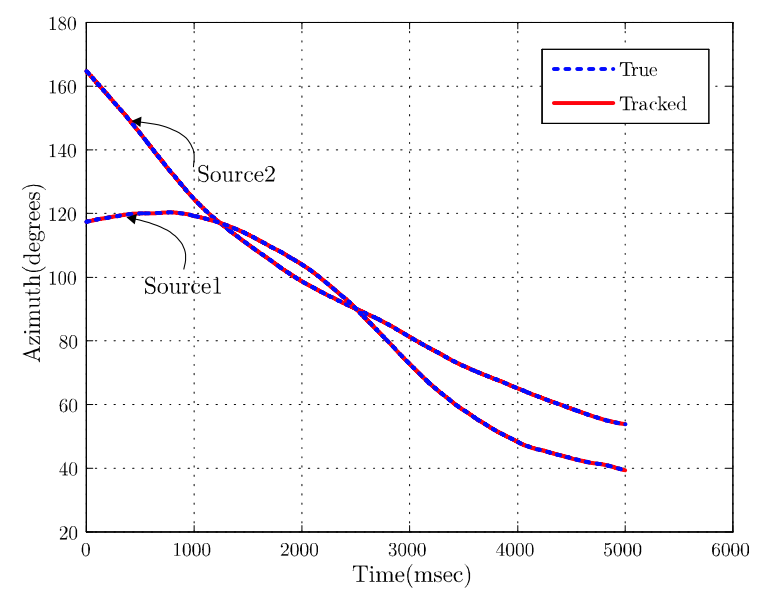

Fig. 16: Azimuth Tracking for the Crossing Situation: True and tracked azimuth angles using the proposed algorithm for two crossing sources for the environment of Figure 14.

also proved that the proposed tracking algorithm can track the locations of the targets with high accuracy and exhibit good performance even in simultaneous crossing situations. Simulation results were presented to demonstrate the ability of the proposed algorithm to track the locations and velocities of the targets with high accuracy in various scenarios.

\section{REFERENCES}

[1] A. Roy, S. Das, and A. Misra, "Exploiting information theory for adaptive mobility and resource management in future cellular networks," IEEE Wireless Communications, vol. 11, no. 4, pp. 59 - 65, aug. 2004.

[2] A. Farina and S. Pardini, "Survey of radar data-processing techniques in air-traffic-control and surveillance systems," IEE Proceedings F Communications, Radar and Signal Processing, vol. 127, no. 3, pp. 190 -204 , june 1980 


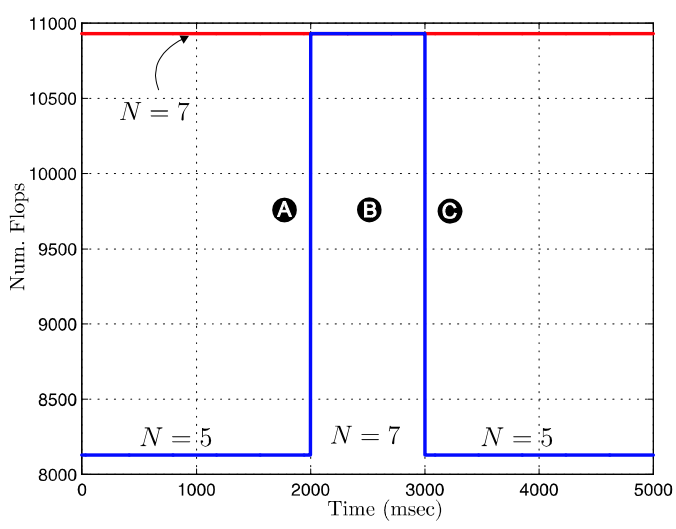

Fig. 17: Computational Complexity for the Crossing Situation: Computational complexity of the proposed algorithm for two crossing sources for the environment of Figure 14. The number of array elements changes at time 2 s from $N=5$ to $N=7$ and then at time $3 \mathrm{~s}$ back to $N=5$ again. Also shown is the complexity when $N$ is fixed at 7 .

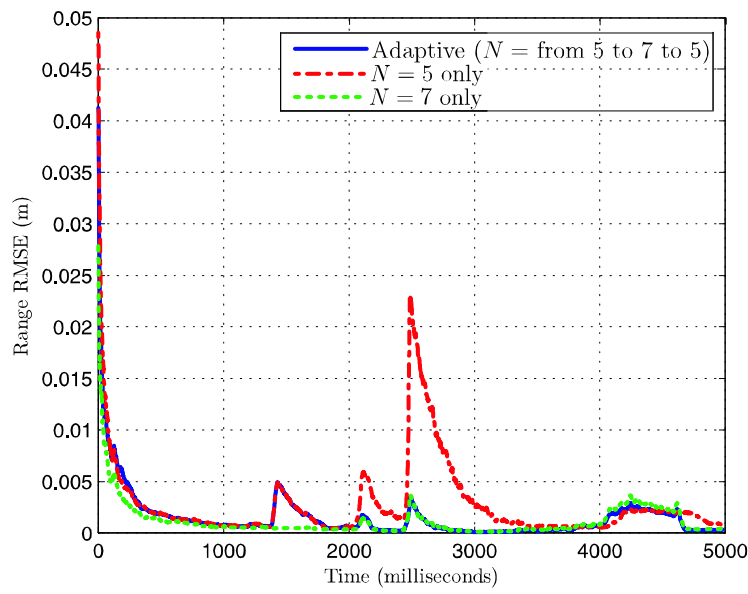

Fig. 18: Range Tracking RMSE Performance: RMSE performance of the range tracking using the "adaptive" $N$ compared with tracking using only $N=5$ and only $N=7$ for two crossing sources for the environment of Figure 14.

[3] R. Kenefic and P. Goulette, "Sensor netting via the discrete time extended kalman filter," IEEE Transactions on Aerospace and Electronic Systems, vol. AES-17, no. 4, pp. 482 -489, july 1981.

[4] D. Balakrishnan and A. Nayak, "An efficient approach for mobile asset tracking using contexts," IEEE Transactions on Parallel and Distributed Systems, vol. 23, no. 2, pp. $211-218$, feb. 2012.

[5] S. Tao, V. Manolopoulos, S. Rodriguez, M. Ismail, and A. Rusu, "Hybrid vehicle positioning and tracking using mobile phones," in ITS Telecommunications (ITST), 2011 11th International Conference on, aug. 2011, pp. $315-320$.

[6] A. Oka and L. Lampe, "Distributed target tracking using signal strength measurements by a wireless sensor network," IEEE Journal on Selected Areas in Communications, vol. 28, no. 7, pp. 1006 -1015, Sep. 2010.

[7] W.-L. Yeow, C.-K. Tham, and W.-C. Wong, "Energy efficient multiple target tracking in wireless sensor networks," IEEE Transactions on Vehicular Technology, vol. 56, no. 2, pp. 918 -928, march 2007.

[8] Y. Zhou, P. C. Yip, and H. Leung, "Tracking the Direction-of-Arrival of Multiple Moving Targets by Passive Arrays : Algorithm," October, vol. 47, no. 10, pp. 2655-2666, 1999.

[9] V. Cevher and J. McClellan, "General direction-of-arrival tracking with acoustic nodes," IEEE Transactions on Signal Processing, vol. 53, no. 1, pp. 1-12, Jan. 2005.

[10] M. Orton and W. Fitzgerald, "A Bayesian approach to tracking multiple targets using sensor arrays and particle filters," IEEE Transactions on Signal Processing, vol. 50, no. 2, pp. 216-223, 2002.

[11] P. Smith and G. Buechler, "A branching algorithm for discriminating and tracking multiple objects," IEEE Transactions on Automatic Control, vol. 20, no. 1, pp. 101-104, 1975.

[12] C. Morefield, "Application of 0-1 integer programming to multitarget tracking problems,' IEEE Transactions on Automatic Control, vol. 22, no. 3, pp. 302-312, 1977.

[13] Y. Bar-Shalom and E. Tse, "Tracking in a cluttered environment with probabilistic data association," Automatica, vol. 11, pp. 451-460, 1975

[14] T. Kirubarajan and Y. Bar-Shalom, "Probabilistic data association techniques for target tracking in clutter," in Proceedings of the IEEE, vol. 92, no. 3, 2004, pp. 536-557.

[15] S. Park, C. Ryu, and K. Lee, "Multiple target angle tracking algorithm using predicted angles," IEEE Transactions on Aerospace and Electronic Systems, vol. 30, no. 2, pp. 643-648, 1994.

[16] C. Rao, C. Sastry, and B. Zhou, "Tracking the direction of arrival of multiple moving targets," IEEE Transactions on Signal Processing, vol. 42, no. 5, pp. $1133-1144,1994$.

[17] P. Chavali and A. Nehorai, "Scheduling and Power Allocation in a Cognitive Radar Network for Multiple-Target Tracking," IEEE Transactions on Signal Processing, vol. 60, no. 2, pp. 715-729, 2012.

[18] R. Niu and R. Blum, "Target Localization and Tracking in Noncoherent Multiple-Input Multiple-Output Radar Systems," IEEE Transactions on Aerospace and Electronic Systems, vol. 48, no. 2, pp. 1466-1489, 2012.

[19] D. Henke, C. Magnard, M. Frioud, D. Small, E. Meier, and M. Schaepman, "Moving-target tracking in single-channel wide-beam sar," IEEE Transactions on Geoscience and Remote Sensing, vol. 50, no. 11, pp. 4735-4747, Nov 2012

[20] J. Hu, X. li Ding, Z. wei Li, J. jun Zhu, Q. Sun, and L. Zhang, "Kalmanfilter-based approach for multisensor, multitrack, and multitemporal insar," IEEE Transactions on Geoscience and Remote Sensing, vol. 51, no. 7, pp. 4226-4239, July 2013.

[21] K. Luo and A. Manikas, "Superresolution multitarget parameter estimation in mimo radar," IEEE Transactions on Geoscience and Remote Sensing, vol. 51, no. 6, pp. 3683-3693, June 2013

22] A. Haimovich, R. Blum, and L. Cimini, "Mimo radar with widely separated antennas," IEEE Signal Processing Magazine, vol. 25, no. 1, pp. 116-129, 2008.

[23] K. Bell and H. Van Trees, "Posterior Cramér-Rao bound for tracking target bearing," in 13th Annu. Workshop Adaptive Sens. Array Process.(ASAP), Lincoln, Lab., Lexington, MA, 2005.

[24] X. Zhang, P. Willett, and Y. Bar-Shalom, "Dynamic Cramer-Rao bound for target tracking in clutter," IEEE Transactions on Aerospace and Electronic, vol. 41, no. 4, pp. 1154 - 1167, 2005.

[25] M. Hernandez, B. Ristic, and A. Farina, "A performance bound for maneuvering target tracking using best-fitting Gaussian distributions,' International Conference on Information Fusion, vol. 1, 2005.

[26] M. Simandl, J. Kralovec, and P. Tichavsky, "Filtering predictive and smoothing Cramer Rao bounds for discrete-time nonlinear dynamic systems," Automatica, vol. 37, no. 11, pp. 1703 - 1716, 2001.

[27] H. Van Trees, Detection, Estimation, and Modulation Theory, Part 1 New York, NY: John Wiley \& Sons New York, 1968.

[28] L. Huang and A. Manikas, "Space-doppler parameter estimation for space-time-doppler reception in time-varying multipath ds-cdma systems," 14th International Conference on Digital Signal Processing, vol. 2, pp. 1309-1312, 2002.

[29] M. Wax and T. Kailath, "Detection of signals by information theoretic criteria," IEEE Transactions on Acoustics, Speech and Signal Processing, vol. 33, no. 2, pp. $387-392$, apr 1985 .

[30] H. Akaike, "Information theory and an extension of the maximum likelihood principle," 2nd International Symposium on Information Theory, pp. 267-281, 1973.

[31] J. Rissanen, "Modeling by shortest data description," Automatica, vol. 14 , pp. 465-471, 1978.

[32] A. Manikas, Y. Kamil, and M. Willerton, "Source Localization using Large Aperture Sparse Arrays," IEEE Transactions on Signal Processing, vol. 60, no. 12, pp. 1-13, Dec. 2012.

[33] S. Kay, Fundamentals of statistical signal processing: estimation theory. Prentice Hall, 1993.

[34] V. Cevher, R. Velmurugan, and J. H. McClellan, "Acoustic Multitarget Tracking Using Direction-of-Arrival Batches," IEEE Transactions on Signal Processing, vol. 55, no. 6, pp. 2810-2825, Jun. 2007. 
[35] P. Tichavsky, C. Muravchik, and A. Nehorai, "Posterior Cramer Rao bounds for discrete-time nonlinear filtering," IEEE Transactions on Signal Processing, vol. 46, no. 5, pp. 1386-1396, 1998.

[36] R. Horn and C. Johnson, Matrix analysis. Cambridge University Press, 1990.

[37] P. Stoica and A. Nehorai, "Performance study of conditional and unconditional direction-of-arrival estimation," IEEE Transactions on Acoustics, Speech, and Signal Processing, vol. 38, no. 10, pp. 17831795, 1990. 